\title{
Caracterización de las asociaciones de cocolitóforos en las cuencas offshore del Pacífico colombiano
}

\section{Characterization of coccolithophore assemblages in the offshore basins of the Colombian Pacific}

\author{
Esteban Osorio Gómez ${ }^{1-2 *}$; Diego Vallejo Hincapie ${ }^{1-2}$; Daniel Rincón Martinez ${ }^{3}$, Andrés Pardo Trujillo ${ }^{1-2}$; \\ $\begin{array}{llll}\text { (D) } 0000-0002-7074-7287 & \text { (D) } 0000-0002-0743-0906 & \text { (D) } 0000-0002-5684-2130 & \text { (D) } 0000-0001-9678-6818\end{array}$ \\ Sandra Restrepo Acevedo ${ }^{3}$ y Raúl Trejos Tamayo ${ }^{1-2}$ \\ (iD) $0000-0002-1904-0892 \quad$ (ID) $0000-0003-1093-8492$
}

1. Instituto de Investigaciones en Estratigrafía IIES, Universidad de Caldas, Manizales, Colombia. * osorio.gomez.esteban@gmail.com
2. Departamento de Ciencias Geológicas Universidad de Caldas. Calle $65 N^{\circ} 26-10$, Manizales, Colombia.
3. Centro de Innovación y Tecnología Instituto Colombiano del Petróleo, ICP-Ecopetrol, km 7 vía Piedecuesta, Santander, Colombia.

RESUMEN

$\mathrm{E}$ 1 presente estudio fue llevado a cabo en los cocolitóforos extraídos de 39 piston core perforados en el offshore de las cuencas Chocó y Tumaco, en el Pacífico colombiano. Los análisis cualitativos y cuantitativos mostraron cambios en las abundancias relativas y en el grado de preservación de los 20 taxones identificados. Los sedimentos examinados en ambas cuencas revelaron tener una asociación de cocolitos $>2 \%$ constituida por Gephyrocapsa oceanica, Gephyrocapsa muellerae, Gephyrocapsa $<3 \mu \mathrm{m}$, Emiliania huxleyi, Calcidiscus leptoporus y Helicosphaera carteri. Con una abundancia más baja $(<2 \%)$, se observaron Ceratolithus spp., Coccolithus pelagicus, Florisphaera profunda, Helicosphaera princei, Helicosphaera sellii, Helicosphaera wallichii y Pontosphaera spp., junto con formas retrabajadas de Reticulofenestra spp., Sphenolithus spp. y Discoaster spp. La identificación de E. huxleyi dentro de la asociación indica que la edad de estos sedimentos no debe ser más antigua que la biozona NN21 del Pleistoceno Medio (Ioniano), aunque formas retrabajadas de edad Mioceno-Plioceno también fueron vistas en los sedimentos. Las estimaciones del número de cocolitos por gramo (cc/g) indicaron que la abundancia promedio de la Cuenca Chocó fue $5,7 \times 10^{6} \mathrm{cc} / \mathrm{g}$, la cual es menor que la Cuenca Tumaco con $1,2 \times 10^{7} \mathrm{cc} / \mathrm{g}$. El análisis estadístico de redundancia (RDA) sugiere que la distancia a la línea de costa es la variable determinante que controla estas diferencias de abundancias relativas y distribución de los cocolitos en ambas cuencas.

PALABRAS CLAVES: Cocolitóforos, Pacífico colombiano, Cuenca Chocó, Cuenca Tumaco, Cuaternario.

\section{ABSTRACT}

$\mathrm{T}$ This study was conducted on coccolithophores recovered from 39 piston-core samples taken offshore Chocó and Tumaco basins, on the Colombian Pacific. Qualitative and quantitative analyses of the coccoliths showed changes in the relative abundances and the state of preservation in the two basins. The examined sediments were characterized by the coccoliths Gephyrocapsa oceanica, Gephyrocapsa muellerae, Gephyrocapsa $<3 \mu \mathrm{m}$, Emiliania huxleyi, Calcidiscus leptoporus and Helicosphaera carteri, which presented abundances higher than $2 \%$. We also identified a minority assemblage $(<2 \%)$ constituted by Ceratolithus spp., Coccolithus pelagicus, Florisphaera profunda, Helicosphaera princei, Helicosphaera sellii, Helicosphaera wallichii and Pontosphaera spp. together with reworked specimens of Reticulofenestra spp,Sphenolithus spp. and Discoaster spp. The recovery of E. huxleyi as part of the assemblage indicates that the studied sediments are younger than the biozone NN21, covering an age range of Middle Pleistocene (Ionian). The number of coccoliths per gram (cc/g) was calculated, demonstrating an average of $5.7 \times 10^{6} \mathrm{cc} / \mathrm{g}$ and $1.2 \times 10^{7} \mathrm{cc} / \mathrm{g}$ for Chocó and Tumaco basins, respectively. In order to interpret the causes of this variance, we performed a multivariate redundancy analysis (RDA), showing that the distance to the coastline is the controlling factor of the fluctuations of the relative abundances and distribution of the coccoliths in both basins.

KEYWORDS: Coccolithophores, Colombian Pacific, Chocó basin, Tumaco basin, Quaternary. 


\section{INTRODUCCIÓN}

Los cocolitóforos son microalgas marinas, unicelulares, pertenecientes a la división Haptophyta, que normalmente presentan una estructura externa compuesta de diminutas placas $(<30 \mu \mathrm{m})$ de carbonato de calcio conocidas como cocolitos (Jordan et al., 1995). Una vez que el microorganismo muere, los cocolitos se hunden en la columna de agua, ya sea en forma de pellets fecales o fragmentos desarticulados incluidos en la "nieve marina", alcanzando el fondo oceánico donde pasan a constituir parte del sedimento (Young et al., 2003; Flores y Sierro, 2007). Una vez que se encuentran en las rocas, los cocolitóforos se incluyen en el grupo de nanofósiles calcáreos, incluyendo algunas formas similares que han sido observadas en sedimentos tan antiguos como el Triásico tardío (Bown, 1998). Los cocolitóforos han sido observados desde los océanos polares hasta los mares tropicales como parte del fitoplancton marino, habitando exclusivamente en la zona fótica (Flores y Sierro, 2007). Debido a su alta abundancia y capacidad de llevar a cabo procesos como la fotosíntesis y la calcificación, los cocolitóforos juegan un papel fundamental en la regulación del sistema océanoatmósfera a través de los ciclos biogeoquímicos del $\mathrm{CO}_{2}$ del planeta (Thierstein y Young, 2004). El estudio de estos microorganismos y su registro fósil ha ganado importancia en investigaciones paleoclimáticas y paleoceanográficas, ya que se ha demostrado su alta susceptibilidad ante los cambios de temperatura superficial marina, disponibilidad de nutrientes, luminosidad, y salinidad superficial; asimismo su uso como herramienta bioestratigráfica es habitual y de gran utilidad (Giraudeau, 1992; Flores et al., 1997, 1999, 2000; Balch, 2004; Antunes, 2007; Saavedra-Pellitero et al., 2010, 2011; Poulton et al., 2017). El océano Pacífico ecuatorial colombiano, lugar donde se desarrolla este trabajo, presenta características oceanográficas particulares, entre las que se destacan: 1) Sus altas tasas de precipitación (2000 a 12700 $\mathrm{mm} / \mathrm{año}$ ) que alimentan en alta medida los cauces de los ríos San Juan, Patía y Mira, entre otros. Estos afluentes de agua dulce arrastran una gran cantidad de sedimentos calculado en $35 \times 10^{9} \mathrm{~m}^{3} / \mathrm{año}$, los cuales afectan considerablemente las propiedades físico-químicas de las aguas superficiales marinas tales como salinidad, turbidez, concentración de nutrientes y temperatura (Poveda y Mesa, 2000; Restrepo y Kjerfve, 2000; CCCP, 2002; Betancur y Martínez, 2003; Gómez y Martínez, 2005; López y Costeros, 2006; Restrepo, 2006; Restrepo y López, 2008; Patarroyo y Martínez 2013,

\section{INTRODUCTION}

Coccolithophores are unicellular, marine microalgae belonging to the Haptophyta Division, which normally have an external structure composed of tiny calcium carbonate plates $(<30 \mu \mathrm{m})$ known as coccoliths (Jordan et al., 1995). Once the microorganism dies, the coccoliths sink into the water column, either in the form of fecal pellets or disarticulated fragments included in the "marine snow", reaching the ocean floor where they become part of the sediment (Young et al., 2003; Flores and Sierro, 2007). Once found in rocks, coccolithophores are included in the group of calcareous nannofossils, including some similar forms that have been observed in sedimentary record as old as the late Triassic (Bown, 1998). Coccolithophores have been observed from the polar oceans to the tropical seas as part of marine phytoplankton, living exclusively in the photic zone (Flores and Sierro, 2007). Due to their high abundance and capacity for processes such as photosynthesis and calcification, coccolithophores play a fundamental role in the regulation of the ocean-atmosphere system through the planet's biogeochemical $\mathrm{CO}_{2}$ cycles (Thierstein and Young, 2004). The study of these microorganisms and their fossil record has gained importance in paleoclimatic and paleoceanographic research, as their high susceptibility to changes in sea surface temperature, nutrient availability, luminosity, and surface salinity has been demonstrated. Likewise, their use as a biostratigraphic tool is common and very useful (Giraudeau, 1992; Flores et al.., 1997, 1999, 2000; Balch, 2004; Antunes, 2007; Saavedra-Pellitero et al., 2010, 2011; Poulton et al., 2017). The Colombian equatorial Pacific Ocean, where this work is carried out, has particular oceanographic characteristics, among which the following stand out: 1) Its high precipitation rates $(2,000$ to $12,700 \mathrm{~mm} /$ year) that to a great extent feed the channels of the rivers San Juan, Patía, and Mira, among others. These freshwater tributaries carry a large amount of sediment calculated at $35 \times 10^{9} \mathrm{~m}^{3} /$ year, which considerably affects the physical-chemical properties of marine surface waters such as salinity, turbidity, nutrient concentration and temperature (Poveda and Mesa, 2000; Restrepo and Kjerfve, 2000; CCCP, 2002; Betancur and Martínez, 2003; Gómez and Martínez, 2005; López and Costeros, 2006; Restrepo, 2006; Restrepo and López, 2008; Patarroyo and Martínez 2013, 2016); 2) The seasonal dynamics of oceanic upwelling, caused by low-level winds that cross the Panamanian 
2016); 2) La dinámica estacional de surgencia oceánica, ocasionada por corrientes de bajo nivel que atraviesan el istmo panameño desde enero hasta marzo, producen un enfriamiento de las aguas superficiales y un incremento en la concentración de nutrientes debido al ascenso de aguas más profundas hacia la superficie (D'Croz y O'Dea, 2007, 2009).

El presente estudio tiene como objetivo determinar la composición y distribución de las asociaciones cuaternarias de cocolitos analizados en los 39 core tops extraídos del fondo marino de los departamentos de Chocó y Nariño (Figura 1). Esta información permitirá establecer un ámbito de tiempo y conocer el patrón de distribución de las comunidades de cocolitóforos que habitan en estas aguas marinas tropicales cercanas a la costa del Pacífico colombiano. Adicionalmente, esta información es un punto de partida para las bases de datos que pretendan analizar la composición del nanoplancton calcáreo en el territorio colombiano.

\section{ÁREA DE ESTUDIO}

El Pacífico tropical oriental es una región caracterizada por la confluencia de corrientes marinas provenientes del norte (Corriente de California) y del sur (Corriente de Perú), dando origen a una zona fronteriza conocida como Frente Ecuatorial, la cual limita la circulación entre aguas superficiales templadas del norte y ecuatoriales frías de alta salinidad del sur (Wyrtki, 1965, 1966; Pak y Zaneveld, 1974; Okada, 1983; Fiedler y Talley, 2006). El océano Pacífico en Colombia se caracteriza por tener una importante dinámica océano- atmósfera en la que convergen los vientos alisios del norte y del sur, controlados por el desplazamiento de la Zona de Convergencia Intertropical (Wyrtki, 1965, 1966; Pak y Zaneveld, 1974; Fiedler y Talley, 2006). De acuerdo con Knauss (1960), Wyrtki (1966) y Fiedler y Talley (2006), las corrientes oceánicas que influyen en la dinámica superficial del Pacífico colombiano son: (a) Contracorriente Ecuatorial (CCE) de aguas tropicalessubtropicales con alta concentración de nutrientes $\left(>25^{\circ} \mathrm{C} \mathrm{y}\right.$ $<34$ UPS); (b) Corriente Surecuatorial (CSE) que transporta considerables volúmenes de agua cálida hacia el oeste, y su límite hacia el norte es con la CCE; y (c) Corriente del Perú (CP), también conocida como Corriente de Humboldt, caracterizada por sus aguas frías templadas y eutróficas (15 a $28^{\circ} \mathrm{C}$ y $>36$ psu). Por otra parte, entre las corrientes subsuperficiales que circulan en el Pacífico colombiano se encuentran la Subcorriente Ecuatorial o de Cromwell y la
Isthmus from January to March, produce a cooling of surface waters and an increase in nutrient concentration due to the ascent of deeper waters to the surface (D'Croz and O'Dea, 2007, 2009).

The objective of this study is to determine the composition and distribution of the quaternary assemblages of coccoliths analyzed in the 39 core tops extracted from the seabed in the departments of Chocó and Nariño (Figure 1). This information will establish a time range and examine the distribution pattern of the coccolithophores communities that inhabit these tropical marine waters near the Colombian Pacific coast. Additionally, this information is a starting point for databases through which to analyze the composition of calcareous nanoplankton in the Colombian territory.

\section{STUDY AREA}

The eastern tropical Pacific is an area characterized by the confluence of marine currents from the north (California Current) and the south (Peru Current), giving rise to a border zone known as the Equatorial Front, which limits circulation between temperate surface waters of the north and cold highly saline equatorial waters of the south (Wyrtki, 1965, 1966; Pak and Zaneveld, 1974; Okada, 1983; Fiedler and Talley, 2006). The Pacific Ocean in Colombia is characterized by an important ocean-atmosphere dynamic in which the trade winds of the north and south converge, controlling the displacement of the Intertropical Convergence Zone (Wyrtki, 1965, 1966; Pak and Zaneveld, 1974; Fiedler and Talley, 2006). According to Knauss (1960), Wyrtki (1966) and Fiedler and Talley (2006), the oceanic currents that influence the surface dynamics of the Colombian Pacific are: (a) the Equatorial Countercurrent (ECC) from tropicalsubtropical waters with high nutrient concentration ( $>25$ ${ }^{\circ} \mathrm{C}$ and $<34 \mathrm{psu}$ ); (b) the Equatorial Under Current (SEC) which transports considerable volumes of warm water to the west, and its northern boundary to the west is with the ECC; and (c) the Peru Current (PC), also known as the Humboldt Current, characterized by its cold temperate and eutrophic waters ( 15 to $28{ }^{\circ} \mathrm{C}$ and $>36 \mathrm{psu}$ ). On the other hand, among the subsurface currents circulating in the Colombian Pacific are the Equatorial or Cromwell Under Current and the Intermediate Equatorial Current (Restrepo and Correa, 2002). In addition to these, two currents described by Restrepo and López (2008) and Rodríguez-Rubio et al. (2008) on the continental margin of the Colombian Pacific 
Corriente Ecuatorial Intermedia (Restrepo y Correa, 2002). En adición a éstas, se registran dos corrientes descritas por Restrepo y López (2008) y Rodríguez-Rubio et al. (2008), en el margen continental del Pacífico colombiano, que son: la Corriente Subsuperficial de Colombia (CSC) y la Contracorriente del Chocó (CCC). are reported as follows: the Colombian Subsurface Current (CSC) and the Chocó Counter Current (CCC).

Salinities and temperatures in the Colombian Pacific Ocean behave inversely. In regions with high temperatures, salinity tends to be low (CCCP, 2002). The sea surface temperature has oscillated between 24.5 and $29.8^{\circ} \mathrm{C}$ during

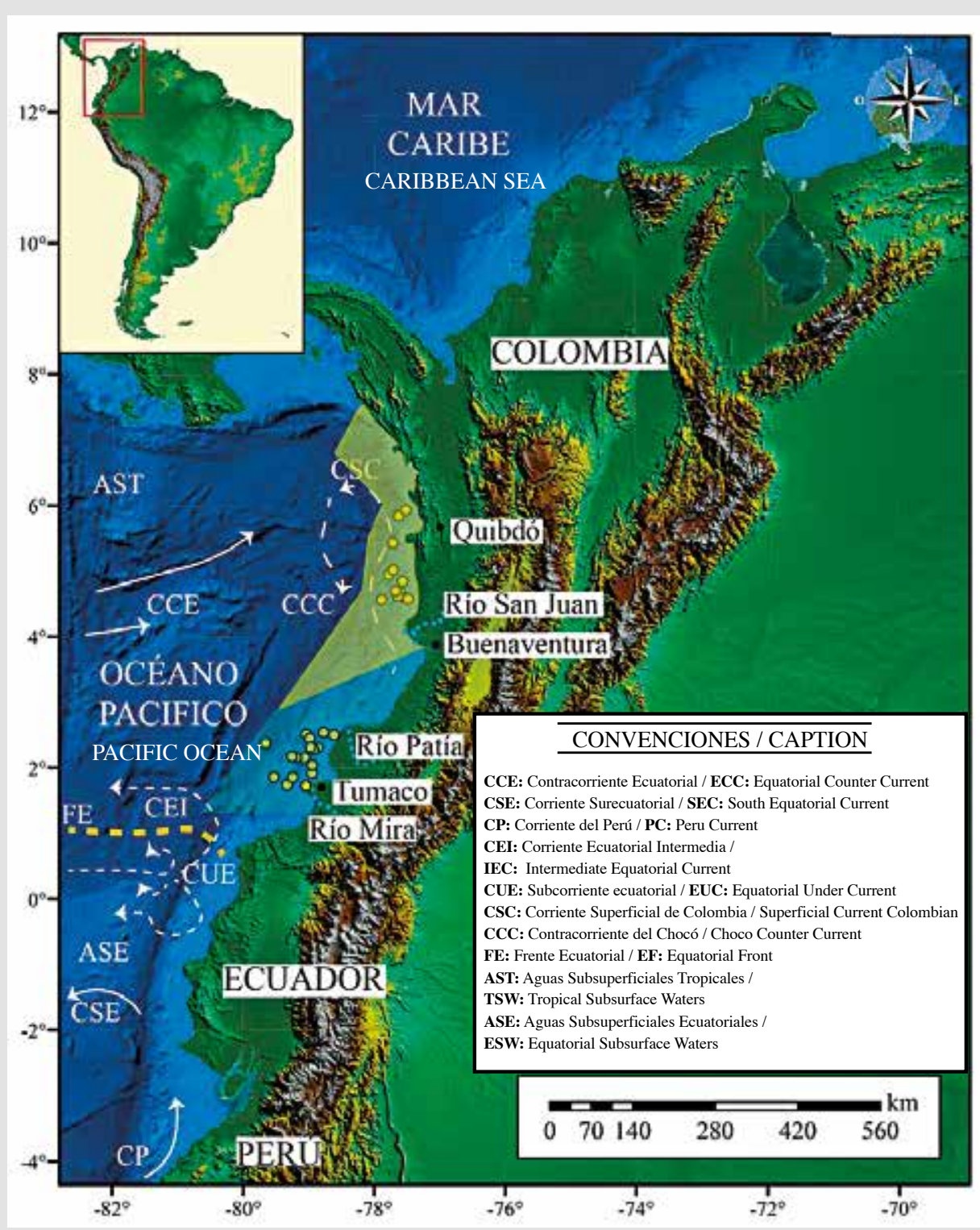

Figura 1. Algunas características de las corrientes oceánicas en el océano Pacíico colombiano. Las corrientes superficiales están representadas por las líneas continuas de color blanco; las líneas discontinuas en color blanco indican las corrientes subsuperficiales; el Frente Ecuatorial con líneas discontinuas color naranja. También se representan las aguas superficiales tropicales y ecuatoriales. Los polígonos amarillo y azul delimitan las áreas de las Cuencas Chocó y Tumaco respectivamente, y los puntos amarillos indican la localización de los piston core. Mapa realizado integrando la información registrada en Wyrtki (1966), Restrepo y Correa (2002), Fiedler y Talley (2006), Restrepo y López (2008) y Rodríguez-Rubio et al. (2008).
Figure 1. Some characteristics of ocean currents in the Colombian Pacific Ocean. The Surface Currents are represented by the continuous white lines; the discontinuous white lines indicate the Subsurface Currents. The Equatorial Front is represented by discontinuous orange lines. Tropical and Equatorial Surface Waters are also represented. The yellow and blue polygons delimit the areas of the Chocó and Tumaco Basins respectively, and the yellow dots indicate the location of the core pistons. Map drawn up by integrating the information reported in Wyrtki (1966), Restrepo and Correa (2002), Fiedler and Talley (2006), Restrepo and López (2008) and Rodríguez-Rubio et al. (2008). 
Las salinidades y temperaturas en elocéano Pacífico colombiano se comportan de forma inversa, observándose que en las regiones donde existe una temperatura alta, las salinidades tienden a ser bajas (CCCP, 2002). La temperatura marina superficial ha oscilado entre 24,5 y 29,8 ${ }^{\circ} \mathrm{C}$ durante los últimos 100 años (Málikov y Villegas, 2005; NOAA, 2017), siendo mayor en las regiones proximales a la costa y menor hacia las más distales (Figura 2). Las aguas superficiales tienden a presentar una temperatura superficial más alta en la región de Chocó que en Tumaco, esto se debe principalmente a que la Contracorriente del Chocó transporta aguas cálidas provenientes del norte (CCCP, 2002). Este patrón se relaciona estrechamente con los cambios de salinidad en el Pacífico colombiano, con ámbitos en la costa entre 30 y 32,8 UPS y valores más altos alrededor de 35 psu a medida que se aleja hacia el sur y occidente (CCCP, 2002) (Figura 2). the last 100 years (Málikov and Villegas, 2005; NOAA, 2017), and is warmer in areas close to the coast and cooler towards more distal regions (Figure 2). Surface waters tend to have a higher surface temperature in the Chocó region than in Tumaco region, mainly because the Chocó Countercurrent transports warm waters from the north (CCCP, 2002). This pattern is closely related to changes in salinity in the Colombian Pacific, with coastal ranges between 30 and 32.8 PSU and higher values around 35 PSU as it moves south and west (CCCP, 2002) (Figure 2).

\section{MATERIALS AND METHODS}

Between November 25 and December 11, 2009, and commissioned by Ecopetrol S.A., the company GEMSHRT together with DIMAR carried out a sampling process of the seabed in the technical evaluation areas (TEAs) of

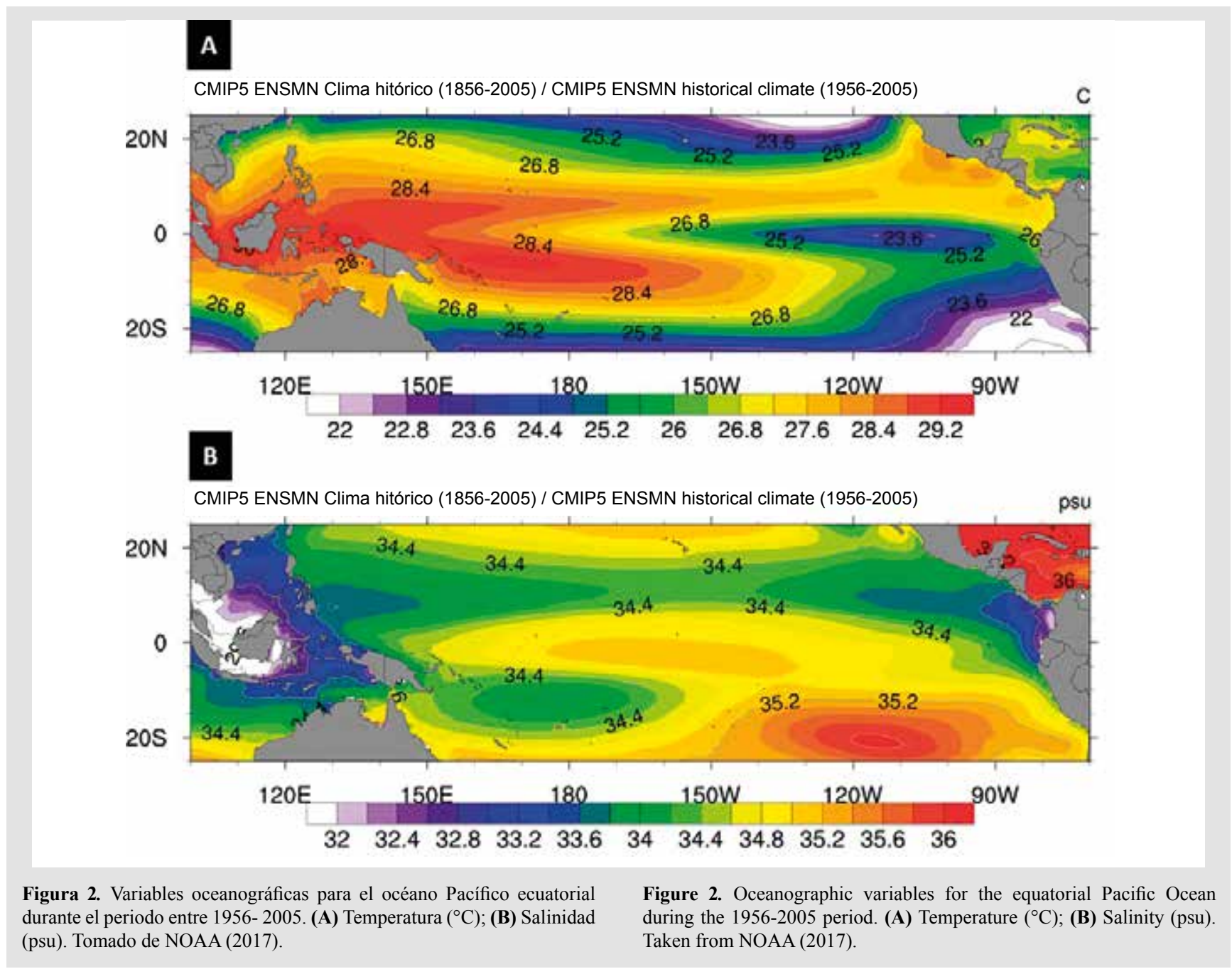




\section{MATERIALES Y MÉTODOS}

Entre el 25 de noviembre y el 11 de diciembre de 2009 la compañía GEMS-HRT y la DIMAR realizaron para Ecopetrol S.A. la toma de muestras del fondo marino en las áreas de evaluación técnica (TEA's) de Merayana (Cuenca Chocó) y Egoro (Cuenca Tumaco), en áreas offshore de los departamentos de Chocó y Nariño respectivamente. La adquisición de 39 piston core se realizó desde la embarcación ARC Providencia a una distancia promedio de $50 \mathrm{~km}$ medida a partir de la línea de costa. Los puntos fueron seleccionados a partir de información sísmica, imágenes batimétricas y la señal de un penetrador de sedimentos de alta frecuencia de 3,5 KHz. Las muestras usadas en este estudio pertenecen a los primeros $11 \mathrm{~cm}$ (techo a base) de los 39 core tops, distribuidas de la siguiente manera: 18 piston core en la Cuenca Tumaco (TEA Egoro) y 21 piston core en la Cuenca Chocó (TEA Merayana) (Figura 3; Tabla 1). Cabe aclarar que los primeros centímetros del material sedimentario recuperado podrían haber experimentado mezcla de sedimentos, como consecuencia del mismo uso de la técnica de piston core.

Preparación de las muestras

El material colectado se preparó en el Laboratorio de Microfósiles Calcáreos del Instituto de Investigaciones en Estratigrafía (IIES) de la Universidad de Caldas, siguiendo la técnica de decantación propuesta por Flores y Sierro (1997). El uso de este método requiere pesar la cantidad de sedimento a decantar y permite obtener una distribución homogénea de los microfósiles en un área de dimensiones conocidas, facilitando así el cálculo de la abundancia de cocolitos por gramo de sedimento a través de la fórmula:

$$
\mathrm{N}=\mathrm{n} \times \mathrm{R}^{2} \times \mathrm{Vx} \mathrm{r}^{-2} \times \mathrm{g}^{-1} \mathrm{x} \mathrm{v}^{-1}
$$

En donde $\mathbf{n}$ es el número de nanofósiles contados en un área determinada, $\mathbf{R}$ el radio de la placa Petri donde se ha decantado la muestra, $\mathbf{V}$ el volumen de agua que se ha añadido al sedimento seco durante la preparación, $\mathbf{r}$ el radio del campo visual del microscopio utilizado en el recuento, $\mathbf{g}$ el peso del sedimento seco y $\mathbf{v}$ el volumen de solución añadido con la micropipeta.

Para el registro fotográfico se usó una cámara Nikon Digital Sight DS-Ri1 con un controlador DS-U2 acoplados a un microscopio petrográfico Nikon Eclipse 50i POL, usando el software Imaging NIS-Elements versión 3.2 de Nikon.
Merayana (Chocó Basin) and Egoro (Tumaco Basin), in offshore zones of the departments of Chocó and Nariño respectively. Thirty-nine piston core samples were collected from the vessel ARC Providencia at an average distance of $50 \mathrm{~km}$ measured from the coastline. The points were selected from seismic information, bathymetric images, and the signal of a $3.5 \mathrm{KHz}$ high frequency sediment penetrator. The samples used in this study belong to the first $11 \mathrm{~cm}$ (base roof) of the 39 core tops, distributed as follows: 18 piston core samples in the Tumaco Basin (TEA Egoro) and 21 piston core samples in the Chocó Basin (TEA Merayana) (Figure 3; Table 1). It should be clarified that the first centimeters of the recovered sedimentary material could have become mixed with sediments, as a result of using the piston core technique.

\section{Sample preparation}

The collected material was prepared in the Calcareous Microfossils laboratory at the Instituto de Investigaciones en Estratigrafía (IIES), Universidad de Caldas, following the decanting technique proposed by Flores and Sierro (1997). This method requires weighing the amount of sediment to be decanted, which then allows a homogeneous distribution of the microfossils in an area of known dimensions, thus enabling the calculation of coccolith abundance per gram of sediment using the formula:

$$
\mathrm{N}=\mathrm{n} \times \mathrm{R}^{2} \times \mathrm{Vx} \mathrm{r}^{-2} \mathrm{xg}^{-1} \mathrm{x} \mathrm{v}^{-1} \text {. }
$$

Where $\mathbf{n}$ is the number of nannofossils counted in a given area, $\mathbf{R}$ is the radius of the Petri dish where the sample was decanted, $\mathbf{V}$ is the volume of water added to the dry sediment during preparation, $\mathbf{r}$ is the radius of the visual field of the microscope used in the count, $\mathbf{g}$ is the weight of the dry sediment, and $\mathbf{v}$ is the volume of solution added with the micropipette.

ANikon Digital Sight DS-Ri1 camera with a DS-U2 controller attached to a Nikon Eclipse 50i POL petrographic microscope was used for the photographic records, using Nikon Imaging NIS-Elements software version 3.2. In order to obtain higher resolution and more detailed photographs, the most abundant samples were prepared for image taking using the Scanning Electron Microscope (SEM). 


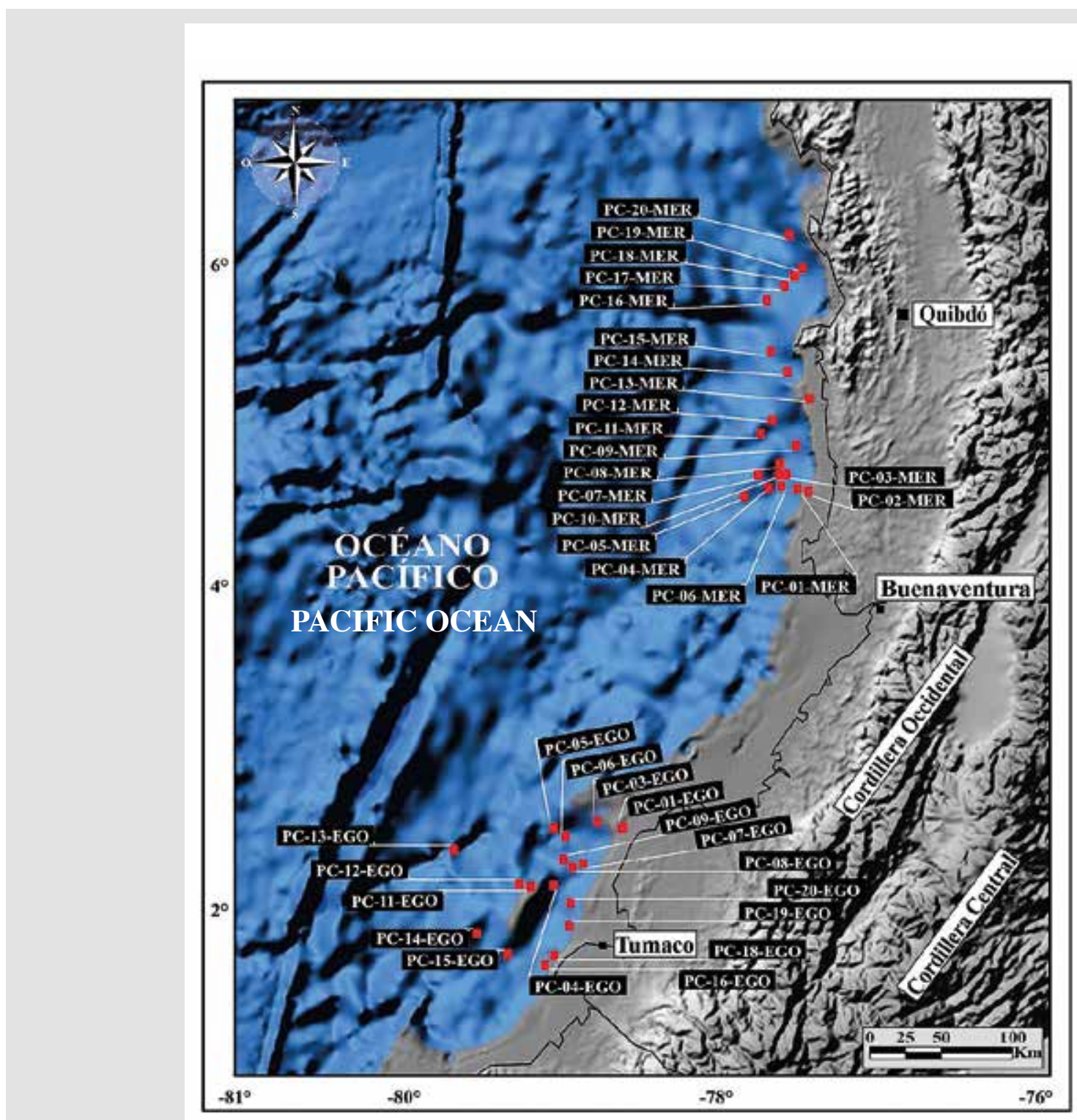

Figura 3. Localización de las zonas de muestreo en las Cuencas Chocó y Tumaco. Nótese la especial localización de la muestra PC-11-EGO sobre un alto topográfico del fondo oceánico.
Figure 3. Location of sampling areas in the Chocó and Tumaco Basins. Note the special location of sample PC-11-EGO on a topographic high of the sea floor.
Con el fin de obtener fotografías de mayor resolución y detalle, las muestras más abundantes fueron preparadas para la toma de imágenes al Microscopio Electrónico de Barrido (SEM).

\section{Análisis microscópicos cualitativos y cuantitativos}

Después de la preparación de 39 placas para el estudio de cocolitóforos se hizo su análisis por medio de un microscopio petrográfico con un aumento de 1000x. Este
Qualitative and quantitative microscopic analyses

To study the coccolithophores, 39 slides were prepared and subsequently analyzed using a petrographic microscope with a magnification of 1000x. This analysis was divided into two parts. Initially, a qualitative estimate of the state of preservation of the coccoliths was carried out according to the criteria established by Roth and Thierstein (1972); Roth (1983); Raffi and Flores (1995) where: (G) Good: The specimens present little or no dissolution and/ 
Tabla 1. Información de las muestras de piston core obtenidos en el Pacífico colombiano. Las muestras que no presentaron ningún contenido de cocolitóforos no se han incluido en esta tabla. *Los valores de las tasas de descarga de sedimentos se han tomado de Restrepo et al. (2000), Restrepo (2006) y Restrepo y López (2008).
Table 1. Information on piston core samples from the Colombian Pacific. Samples that did not contain any coccolithophores are not included in this table. *The values of sediment discharge rates have been taken from Restrepo et al. (2000), Restrepo (2006) and Restrepo and López (2008).

\begin{tabular}{|c|c|c|c|c|c|c|c|c|c|c|}
\hline $\begin{array}{l}\text { Muestra } \\
\text { Sample }\end{array}$ & $\begin{array}{l}\text { Longitud (E) } \\
\text { Longitude }\end{array}$ & $\begin{array}{l}\text { Latitud (N) } \\
\text { Latitude }\end{array}$ & $\begin{array}{l}\text { Cuenca } \\
\text { Basin }\end{array}$ & $\begin{array}{c}\text { Longitud } \\
\text { piston } \\
\text { core }(\mathrm{m}) \\
\text { Longitude } \\
\text { of the piston } \\
\text { core }(\mathrm{m})\end{array}$ & $\begin{array}{c}\text { Profundidad } \\
\text { Agua (m) } \\
\text { Water Depth } \\
\text { (m) }\end{array}$ & $\begin{array}{c}\text { *Descarga } \\
\text { detritos } \\
\text { (Tonelada/ } \\
\text { año) } \\
\text { Discharge } \\
\text { of detritic } \\
\text { material } \\
\text { (Ton/year) }\end{array}$ & $\begin{array}{l}\text { Distancia a } \\
\text { la línea de } \\
\text { costa }(\mathrm{km}) \\
\text { Distance } \\
\text { from } \\
\text { coastline } \\
(\mathrm{km})\end{array}$ & $\begin{array}{c}\text { Cocolitos } \\
\text { por } \\
\text { muestra } \\
\text { Coccoliths } \\
\text { per sample }\end{array}$ & $\begin{array}{l}\text { Abundancia } \\
\qquad(\mathrm{cc} / \mathrm{g})\end{array}$ & $\begin{array}{l}\text { Preservación } \\
\text { Preservation }\end{array}$ \\
\hline PC-01-EGO & -78.6096732333 & 2.5103462051 & $\begin{array}{l}\text { Tumaco } \\
\text { Offshore }\end{array}$ & 2.62 & 76 & 14213160 & 5.86 & 335 & $5.8 \times 10^{6}$ & Buena / Good \\
\hline PC-03-EGO & -78.7683148185 & 2.5486065756 & $\begin{array}{l}\text { Tumaco } \\
\text { Offshore }\end{array}$ & 1.38 & 210 & 14213160 & 25.65 & 149 & $2.2 \times 10^{6}$ & Buena / Good \\
\hline PC-04-EGO & -79.0545701892 & 2.1629215488 & $\begin{array}{l}\text { Tumaco } \\
\text { Offshore }\end{array}$ & 0.85 & 857 & 14213160 & 35.87 & 514 & $1.4 \times 10^{7}$ & Buena / Good \\
\hline PC-05-EGO & -79.0325229342 & 2.5196451204 & $\begin{array}{l}\text { Tumaco } \\
\text { Offshore }\end{array}$ & 1.45 & 653 & 14213160 & 53 & 503 & $1.0 \times 10^{7}$ & Buena / Good \\
\hline PC-06-EGO & -78.9768563495 & 2.4556433403 & $\begin{array}{l}\text { Tumaco } \\
\text { Offshore }\end{array}$ & 1 & 948 & 14213160 & 43.1 & 512 & $1.4 \times 10^{7}$ & Buena / Good \\
\hline PC-07-EGO & -78.8590802792 & 2.3145422459 & $\begin{array}{l}\text { Tumaco } \\
\text { Offshore }\end{array}$ & 1.94 & 768 & 14213160 & 21.93 & 254 & $3.3 \times 10^{6}$ & $\begin{array}{l}\text { Moderada / } \\
\text { Moderate }\end{array}$ \\
\hline PC-08-EGO & -78.9220379326 & 2.2932543049 & $\begin{array}{l}\text { Tumaco } \\
\text { Offshore }\end{array}$ & 1.9 & 814 & 14213160 & 26.25 & 519 & $1.2 \times 10^{7}$ & Buena / Good \\
\hline PC-09-EGO & -78.9682483798 & 2.3189991005 & $\begin{array}{l}\text { Tumaco } \\
\text { Offshore }\end{array}$ & 1.55 & 958 & 14213160 & 34.44 & 520 & $1.3 \times 10^{7}$ & $\begin{array}{l}\text { Moderada / } \\
\text { Moderate }\end{array}$ \\
\hline PC-11-EGO & -79.1825316713 & 2.1638727273 & $\begin{array}{l}\text { Tumaco } \\
\text { Offshore }\end{array}$ & 1.08 & 394 & 14213160 & 50.41 & 395 & $2.0 \times 10^{7}$ & Buena / Good \\
\hline PC-13-EGO & -79.6510112794 & 2.3830263074 & $\begin{array}{l}\text { Tumaco } \\
\text { Offshore }\end{array}$ & 2.24 & 566 & 14213160 & 112.61 & 550 & $2.4 \times 10^{7}$ & Buena / Good \\
\hline PC-14-EGO & -79.5243544789 & 1.8592476226 & $\begin{array}{l}\text { Tumaco } \\
\text { Offshore }\end{array}$ & 0.84 & 797 & 14213160 & 75.76 & 561 & $1.6 \times 10^{7}$ & Buena / Good \\
\hline PC-15-EGO & -79.3228659771 & 1.7461747664 & $\begin{array}{l}\text { Tumaco } \\
\text { Offshore }\end{array}$ & 0.86 & 839 & 14213160 & 40.89 & 595 & $2.4 \times 10^{7}$ & Buena / Good \\
\hline PC-16-EGO & -79.0948435477 & 1.6793223088 & $\begin{array}{l}\text { Tumaco } \\
\text { Offshore }\end{array}$ & 2.87 & 1835 & 14213160 & 8.76 & 557 & $2.0 \times 10^{7}$ & Buena / Good \\
\hline PC-19-EGO & -78.9438990403 & 1.9210978919 & $\begin{array}{l}\text { Tumaco } \\
\text { Offshore }\end{array}$ & 1.6 & 353 & 14213160 & 36.5 & 502 & $8.5 \times 10^{7}$ & Buena / Good \\
\hline PC-20-EGO & -78.9412396271 & 2.0461221603 & $\begin{array}{l}\text { Tumaco } \\
\text { Offshore }\end{array}$ & 1.86 & 664 & 14213160 & 27.26 & 521 & $1.5 \times 107$ & Buena / Good \\
\hline PC-01-MER & -77.4797402623 & 4.5941925792 & $\begin{array}{l}\text { Chocó } \\
\text { Offshore }\end{array}$ & 1.35 & 560 & 16000000 & 22.22 & 508 & $6.4 \times 10^{6}$ & Buena / Good \\
\hline PC-02-MER & -77.6139275567 & 4.702961991 & $\begin{array}{l}\text { Chocó } \\
\text { Offshore }\end{array}$ & 1.98 & 94 & 16000000 & 14.92 & 576 & $1.1 \times 10^{7}$ & Buena / Good \\
\hline PC-03-MER & -77.8844782696 & 4.5831360714 & $\begin{array}{l}\text { Chocó } \\
\text { Offshore }\end{array}$ & 1.44 & 1256 & 16000000 & 29.05 & 314 & $4.6 \times 10^{6}$ & $\begin{array}{c}\text { Moderada / } \\
\text { Moderate }\end{array}$ \\
\hline PC-05-MER & -77.6402657024 & 4.634085925 & $\begin{array}{l}\text { Chocó } \\
\text { Offshore }\end{array}$ & 5.42 & 1534 & 16000000 & 60.75 & 518 & $1.5 \times 10^{7}$ & Buena / Good \\
\hline PC-06-MER & -77.5647760183 & 4.8655732856 & $\begin{array}{l}\text { Chocó } \\
\text { Offshore }\end{array}$ & 1.34 & 1256 & 16000000 & 31.86 & 222 & $2.9 \times 106$ & $\begin{array}{l}\text { Moderada / } \\
\text { Moderate }\end{array}$ \\
\hline PC-09-MER & -77.6714815002 & 4.7172793631 & $\begin{array}{l}\text { Chocó } \\
\text { Offshore }\end{array}$ & 3.42 & 721 & 16000000 & 20.48 & 503 & $6.5 \times 10^{6}$ & $\begin{array}{l}\text { Moderada / } \\
\text { Moderate }\end{array}$ \\
\hline PC-10-MER & -77.7797708887 & 4.9630475484 & $\begin{array}{l}\text { Chocó } \\
\text { Offshore }\end{array}$ & 2.15 & 1189 & 16000000 & 36.47 & 512 & $8.5 \times 10^{6}$ & $\begin{array}{l}\text { Moderada / } \\
\text { Moderate }\end{array}$ \\
\hline PC-11-MER & -77.7103557666 & 5.0410175563 & $\begin{array}{l}\text { Chocó } \\
\text { Offshore }\end{array}$ & 2.98 & 1297 & 16000000 & 43.26 & 334 & $4.4 \times 10^{6}$ & $\begin{array}{l}\text { Moderada / } \\
\text { Moderate }\end{array}$ \\
\hline PC-12-MER & -77.7147988221 & 5.4541612548 & $\begin{array}{l}\text { Chocó } \\
\text { Offshore }\end{array}$ & 3.11 & 1187 & 16000000 & 35.05 & 202 & $2.6 \times 10^{6}$ & $\begin{array}{l}\text { Moderada / } \\
\text { Moderate }\end{array}$ \\
\hline PC-15-MER & -77.5178974509 & 5.958048616 & $\begin{array}{l}\text { Chocó } \\
\text { Offshore }\end{array}$ & 2.87 & 1835 & 16000000 & 17.18 & 129 & $1.7 \times 10^{6}$ & $\begin{array}{l}\text { Moderada / } \\
\text { Moderate }\end{array}$ \\
\hline PC-19-MER & -78.6096732333 & 2.5103462051 & $\begin{array}{l}\text { Chocó } \\
\text { Offshore }\end{array}$ & 2.3 & 1764 & 16000000 & 18.7 & 331 & $4.3 \times 10^{6}$ & $\begin{array}{c}\text { Moderada / } \\
\text { Moderate }\end{array}$ \\
\hline
\end{tabular}


análisis se dividió en dos partes, inicialmente se hizo una estimación cualitativa del estado de preservación de los cocolitos de acuerdo con los criterios establecidos por Roth y Thierstein (1972), Roth (1983) y Raffi y Flores (1995) en donde: (B) Buena: Los ejemplares presentan poca o ninguna disolución y/o recristalización. (B-M) Buena-Moderada: Muestra ligera a moderada disolución y/o recristalización y la identificación de las especies es limitada. (M) Moderada: Los ejemplares presentan disolución moderada y/o recristalización excesiva. (P) Pobre: Los microfósiles presentan una disolución extrema y/o recristalización excesiva.

Posteriormente se procedió a hacer el reconocimiento taxonómico de todos los grupos presentes, siguiendo la guía taxonómica de Young et al. (2003) para las asociaciones de cocolitos del Pleistoceno y las descripciones hechas por Perch-Nielsen (1985) para las formas retrabajadas del Mioceno-Plioceno. Por otra parte, se agruparon bajo el nombre de Gephyrocapsa $<3 \mu \mathrm{m}$ los cocolitos de Gephyrocapsa que se caracterizaron por tener tamaños inferiores a $3 \mu \mathrm{m}$ y que por su diminuto tamaño al microscopio petrográfico no fueron identificados. Una lista de los taxones encontrados en las muestras es presentada en el Apéndice A.

Finalmente se realizó un análisis cuantitativo por medio de dos tipos de recuentos: el primero, un recuento de 500 especímenes por muestra, independientemente del número de campos visuales observados. En muestras con bajo número de cocolitos se contó un máximo de 400 campos visuales. Este método garantiza con un $95 \%$ de confianza que los especímenes con abundancias del $1 \%$ son incluidos dentro de la asociación (Dennison y Hay, 1967; Fatela y Taborda, 2001). El segundo recuento se hizo en 40 campos visuales, buscando exclusivamente aquellas especies que mostraron abundancia $<2 \%$; este recuento se realizó conla finalidad de cuantificar las especies subestimadas o sobreestimadas en el primer conteo (Apéndice B).

\section{Análisis estadísticos}

La matriz de abundancias usada para el análisis estadístico fue creada omitiendo aquellas muestras con una abundancia menor de 100 ejemplares y descartando aquellas especies con abundancias inferiores a 2\% (Schneider et al., 2011). Esto garantiza que las interpretaciones basadas en los resultados estadísticos contienen los taxones más representativos de la asociación (Dennison y Hay, 1967; Fatela y Taborda, 2001; Schneider et al., 2011). or recrystallization. (G-M) Good-Moderate: Light to moderate dissolution and/or recrystallization and species and identification is limited. (M) Moderate: Specimens show moderate dissolution and/or excessive recrystallization. (P) Poor: The microfossils present extreme dissolution and/or excessive recrystallization.

Subsequently, all coccolithophores were taxonomically identified, following the Young et al. taxonomic guide (2003) for Pleistocene coccolith assemblages and the descriptions by Perch-Nielsen (1985) for Miocene-Pliocene reworked forms. Coccoliths of Gephyrocapsa, which were characterized by being smaller than $3 \mu \mathrm{m}$ and not being recognized by the petrographic microscope due to their tiny size, were grouped under the name of Gephyrocapsa $<3 \mu \mathrm{m}$. A list of the taxa found in the samples is presented in Appendix A.

Finally, a quantitative analysis was performed using two types of counts. The first was a count of 500 specimens per sample, regardless of the number of fields of view observed. In samples with a low number of coccoliths, a maximum of 400 visual fields were counted. This method guarantees, to a $95 \%$ confidence level, that specimens with $1 \%$ abundance are included within the assemblage (Dennison and Hay, 1967; Fatela and Taborda, 2001). The second count was conducted in 40 visual fields, looking exclusively for those species that showed abundance $<2 \%$, in order to quantify the species underestimated or overestimated in the first count (Appendix B).

\section{Statistical analysis}

The abundance matrix used for statistical analysis was created by omitting samples with an abundance of less than 100 specimens and discarding species with abundances of less than 2\% (Schneider et al., 2011). This ensures that interpretations based on statistical results contain the most representative taxa of the assemblage (Dennison and Hay, 1967; Fatela and Taborda, 2001; Schneider et al., 2011). Additionally, reworked forms of calcareous nannofossils were also excluded from statistical treatment in order to avoid alterations during processing.

A redundancy analysis (RDA) was performed to summarize the relationships between coccolithophores species and environmental parameters (Zar, 1996; Hammer et al., 2001; Whitlock and Schulter, 2009; Borcard et al., 2011). Counts were standardized from a Hellinger-type transformation to allow low incidence taxa to be weighted in statistical analysis in the R core Team software, (2015) with the Vegan package. The 
Adicionalmente, las formas de nanofósiles calcáreos retrabajadas fueron igualmente excluidas del tratamiento estadístico con la finalidad de evitar alteraciones durante el procesamiento.

Se realizó un análisis de redundancia (RDA) para resumir las relaciones entre las especies de cocolitóforos y los parámetros ambientales (Zar, 1996; Hammer et al., 2001; Whitlock y Schulter, 2009; Borcard et al., 2011). Los conteos se estandarizaron a partir de una transformación tipo Hellinger para permitir que los taxones de baja incidencia se ponderarán en el análisis estadístico en el software $\mathrm{R}$ core Team, (2015) con el paquete Vegan. La significancia de cada parámetro ambiental fue evaluada con base en la longitud del gradiente en el espacio de ordenación y su correlación con los dos ejes principales. La dirección y tamaño de los vectores son indicadores de la influencia de las variables en la distribución de los grupos taxonómicos.

\section{RESULTADOS}

Edad

La presencia de Emiliania huxleyi dentro de la asociación, cuya aparición es situada a partir del Ioniano (Pleistoceno Medio), marca el límite inferior de la biozona NN21 (Martini, 1971). Este bioevento está datado en 290.000 años (Thierstein et al., 1977; Backman et al., 2012). A partir de la asociación de cocolitos encontrada se establece que la edad de estos sedimentos no debe ser más antigua que la biozona NN21 del Ioniano (Pleistoceno Medio).

\section{Asociación y abundancia de cocolitóforos}

Los resultados del análisis microscópico mostraron que de las 39 muestras analizadas 9 resultaron estériles, 8 de la Cuenca Chocó y 1 de la Cuenca Tumaco. El resto de las muestras con contenido de microfósiles permitieron identificar 20 taxones de cocolitóforos en ambas localidades (Figura 4).

Entre los taxones registrados, G. oceanica, $G$. muellerae, Gephyrocapsa $<3 \mu \mathrm{m}$, E. huxleyi, C. leptoporus y $H$. carteri presentaron abundancias relativas mayores a 2\% (Figura 4 E-I; Tabla 2). Por el contrario, especies como Ceratolithus spp., C. pelagicus, F. profunda, H. princei, H. sellii, H. wallichii y Pontosphaera spp. ocuparon abundancias menores a $2 \%$ (Figura $4 \mathrm{~J}-\mathrm{P}$ ). Otro componente común de las asociaciones fueron las formas de nanofósiles retrabajados del Mioceno y el Plioceno, principalmente significance of each environmental parameter was evaluated based on the length of the gradient in the management space and its correlation with the two main axes. The direction and size of the vectors are indicators of the influence of variables on the distribution of taxonomic groups.

\section{RESULTS}

Age

The presence of Emiliania huxleyi - whose appearance dates back to the Ionian (Middle Pleistocene) - in the assemblage marks the lower limit of the biozone NN21 (Martini, 1971). This bioevent dates back 290,000 yrs. (Thierstein et al., 1977; Backman et al., 2012). From the assemblage of coccoliths found, it is established that these sediments should be no older than the biozone NN21 of the Ionian (Middle Pleistocene).

\section{Assemblage and abundance of coccolithophores}

The results of the microscopic analysis showed that out of 39 samples analyzed, 9 samples - 8 from the Chocó Basin and 1 from the Tumaco Basin - were barren. The rest of the samples containing microfossils made it possible to identify 20 taxa of coccolithophores in both localities (Figure 4).

Among the taxa recorded, G. oceanica, $G$. muellerae, Gephyrocapsa $<3 \mu \mathrm{m}$, E. huxleyi, C. leptoporus and $H$. carteri presented relative abundances greater than 2\% (Figure 4 E-I; Table 2). In contrast species such as Ceratolithus spp., C. pelagicus, F. profunda, H. princei, H. sellii, H. wallichii y Pontosphaera spp. presented an abundance of less than $2 \%$ (Figure $4 \mathrm{~J}-\mathrm{P}$ ). Another common component of the assemblages were the reworked Miocene and Pliocene forms of calcareous nannofossils, mainly forms of Reticulofenestra spp. (Figure 4 Q). The sample PC-11-EGO was characterized by having the highest rework values, with a presence of $D$. variabilis, $S$. abies and S. heteromorphus. (Figure 4 R-T).

Although the most modern specimens of coccolithophores were seen in both the Chocó Basin and the Tumaco Basin, there was a contrast in the number of coccoliths per gram (cc/g) calculated for the two regions (Table 1). Similarly, qualitative analyses of the degree of preservation of the coccoliths varied between the two basins, showing that in the Tumaco Basin, overall preservation was good, without 
formas de Reticulofenestra spp. (Figura 4 Q). La muestra PC-11-EGO se caracterizó por presentar los valores más altos de retrabajo, observándose D. variabilis, S. abies, y $S$. heteromorphus. (Figura 4 R-T).

Aunque los especímenes más modernos de cocolitóforos fueron vistos tanto en la Cuenca Chocó como en la Cuenca Tumaco, se evidenció un contraste en cuanto al número de cocolitos por gramo (cc/g) calculados para ambas regiones (Tabla 1). Igualmente, los análisis cualitativos del grado de preservación de los cocolitos variaron entre las dos cuencas, mostrando que en la Cuenca Tumaco la preservación en general fue buena, sin disolución y/o recristalización excesiva; mientras que, los taxones de la Cuenca Chocó comúnmente presentaron una preservación moderada (Tabla 1). No obstante, el reconocimiento a nivel de especies se logró realizar en la mayoría de los ejemplares (Figura 5).

Los cálculos de abundancias de cocolitos por gramo de sedimento evidenciaron que la Cuenca Tumaco presenta los valores más altos, con un promedio de $1,2 \times 10^{7} \mathrm{cc} / \mathrm{g}$. Las abundancias más altas se encuentran en las muestras PC11-EGO, PC-13-EGO, PC-14-EGO, PC-15-EGO y PC-16$\mathrm{EGO}$, con valores alrededor de $2,0 \times 10^{7} \mathrm{cc} / \mathrm{g}$, mientras que las muestras con menor abundancia corresponden a PC-12EGO y PC-18- EGO con valores de 7,9x10 $\mathrm{cc} / \mathrm{g}$ y $6,8 \times 10^{5}$, respectivamente. Los puntos muestreados procedentes de la Cuenca Chocó presentan valores inferiores, con un promedio de $5,7 \times 10^{6} \mathrm{cc} / \mathrm{g}$, donde las muestras PC-02- MER y PC-05MER presentan la mayor abundancia, con valores de $1,1 \times 10^{7}$ y $1,5 \times 10^{7} \mathrm{cc} / \mathrm{g}$ respectivamente. Una menor concentración se observa en las muestras PC-15-MER y PC-17-MER con

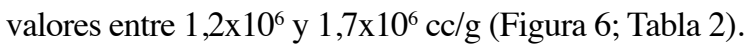

\section{Análisis multivariados}

El análisis de redundancia RDA (Zar, 1996; Hammer et al., 2001; Whitlock y Schulter, 2009; Borcard et al., 2011) proporcionó información significativa sobre la relación entre las abundancias de los cocolitóforos, demostrando que el factor más explicativo y/o correlacionable respecto a la distribución y abundancia de los taxones es fundamentalmente la distancia a la línea de costa (eje 1; 1,4276028; DC) en comparación con la profundidad de la columna de agua (eje 2; 0,5770010; WD) y con la descarga de sedimentos $(-0,4538382$; T.a), (Tabla 3; Figura 7). Estos parámetros explican 74\%, 14\% y $12 \%$ respectivamente de la varianza total. Emiliania huxleyi, Gephyrocapsa $<3 \boldsymbol{\mu m}$ y G. muellerae revelan una tendencia hacia el eje 1 con valores negativos, mientras que el vector de mayor magnitud sobre este eje está dado por H. carteri y excessive dissolution and/or recrystallization; whereas, the taxa of the Chocó Basin commonly presented moderate preservation (Table 1). However, recognition at species level was achieved in most of the specimens (Figure 5).

Calculations of coccoliths abundances per gram of sediment showed that the Tumaco Basin has the highest values, with an average of $1.2 \times 107 \mathrm{cc} / \mathrm{g}$. The highest abundances are found in the samples PC-11-EGO, PC-13EGO, PC-14-EGO, PC-15-EGO and PC-16-EGO, with values of around $2.0 \times 107 \mathrm{cc} / \mathrm{g}$, while the samples with lower abundance correspond to PC-12-EGO and PC-18EGO with values of $7.9 \times 105 \mathrm{cc} / \mathrm{g}$ and $6.8 \times 105$ respectively. The points sampled from the Chocó Basin present lower values, with an average of $5.7 \times 106 \mathrm{cc} / \mathrm{g}$, where samples PC02-MER and PC-05-MER present the highest abundance, with values of $1.1 \times 107$ and $1.5 \times 107 \mathrm{cc} / \mathrm{g}$ respectively. A lower concentration is observed in the samples PC-15-MER and PC-17-MER with values between $1.2 \times 106$ and 1.7x 106 cc/g (Figure 6; Table 2).

\section{Multivariate analyses}

Redundancy analysis RDA (Zar, 1996; Hammer et al., 2001; Whitlock and Schulter, 2009; Borcard et al., 2011) provided significant information on the relationship between coccolithophore abundance, demonstrating that the most explanatory and/or correlated factor with respect to taxon distribution and abundance is related primarily to distance from the coastline (axis $1 ; 1.4276028$; DC) compared to water column depth (axis 2; 0.5770010; WD) and sediment discharge $(-0.4538382$; T.a), (Table 3; Figure 7). These parameters explain $74 \%, 14 \%$, and $12 \%$ respectively of the total variance. E. huxleyi, Gephyrocapsa $<3 \mu \mathrm{m}$ and G. muellerae reveal a trend towards axis 1 with negative values, while the vector of greatest magnitude on this axis is given by H. carteri, and to a lesser extent by C. leptoporus (Figure 7). As for the species G. oceanica, greater correspondence is shown with axis 2 , the vector of this taxon being the closest to said axis, showing preferences for greater depth and affinity for high influence of sediments (Figure 7).

\section{DISCUSSION}

Comparison with other coccolithophore assemblages in the Eastern Tropical Pacific

The coccolith counts indicate that the main assemblage (greater than 2\%) in the Chocó and Tumaco 

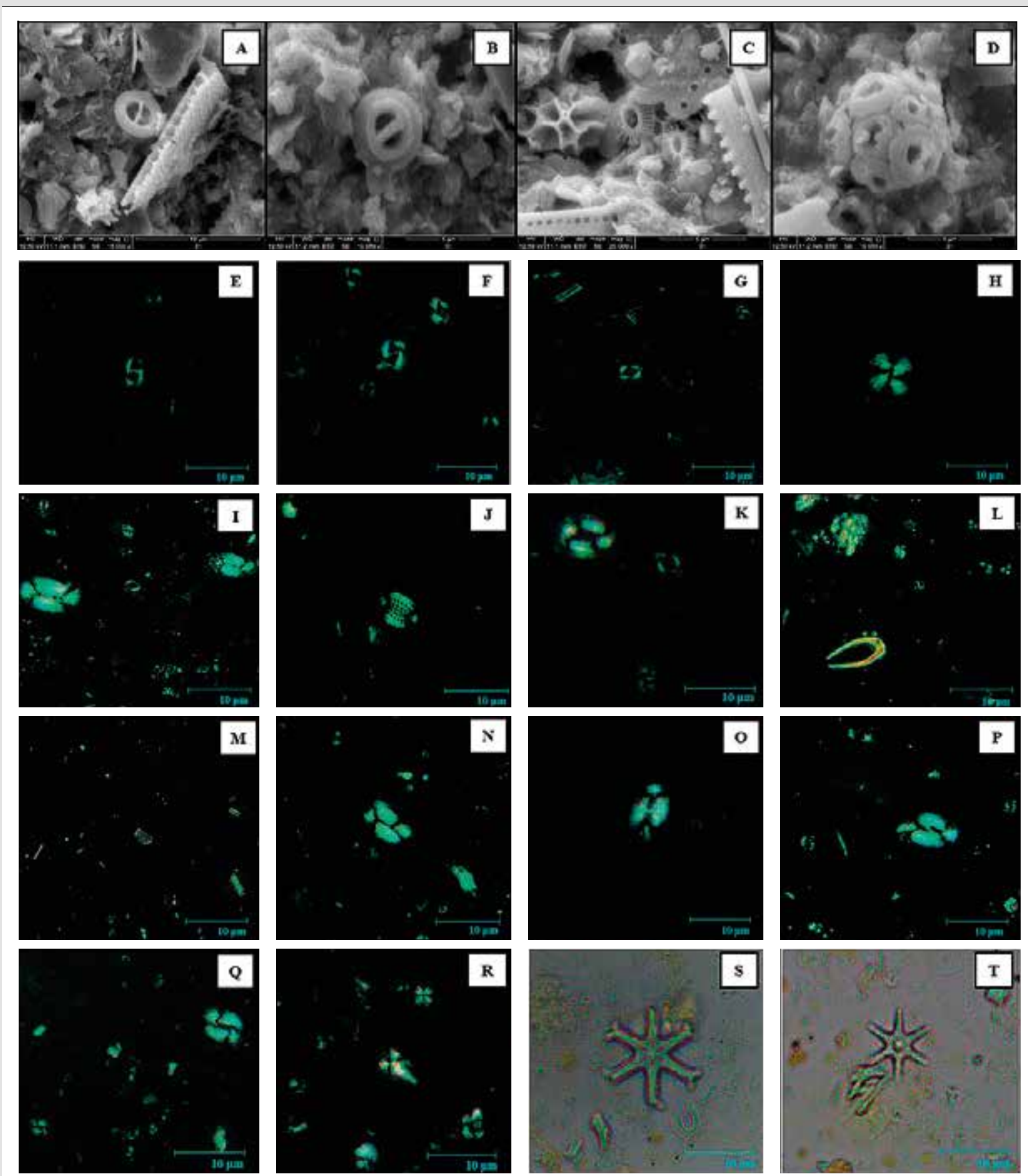

Figura 4. Especies de cocolitos encontrados en las muestras analizadas. Imágenes tomadas bajo el Microscopio Electrónico de Barrido (SEM). A. Gephyrocapsa oceanica $(10 \mu \mathrm{m})$. B. Gephyrocapsa oceanica $(5 \mu \mathrm{m})$. C. Emiliania huxleyi $(5 \mu \mathrm{m})$. D. Coccosphere de Gephyrocapsa spp. $(5 \mu \mathrm{m})$. Imágenes capturadas bajo microscopio petrográfico y ampliadas por 1000x. Nicoles cruzados (campo oscuro). Nicoles paralelos (campo claro). E. Gephyrocapsa oceanica. F. Gephyrocapsa muellerae. G. Emiliania huxleyi. H. Calcidiscus leptoporus. I. Helicosphaera carteri. J. Pontosphaera spp. K. Coccolithus pelagicus. L. Ceratolithus spp. M. Florisphaera profunda. N. Helicosphaera princei. O. Helicosphaera sellii. P. Helicosphaera wallichii. Q. Reticulofenestra pseudoumbilicus. R. Sphenolithus heteromorphus (inferior) y Sphenolithus abies (superior). S-T. Discoaster variabilis.

Figure 4. Species of coccoliths found in the analyzed samples. Images taken under Scanning Electron Microscope (SEM). A. Gephyrocapsa oceanica $(10 \mu \mathrm{m})$. B. Gephyrocapsa oceanica $(5 \mu \mathrm{m})$. C. Emiliania huxleyi $(5 \mu \mathrm{m})$. D. Coccosphere of Gephyrocapsa spp. $(5 \mu \mathrm{m})$.

Images captured under petrographic microscope and enlarged by 1000x. Crossed Nicol (dark field). Parallel Nicol (light field). E. Gephyrocapsa oceanica. F. Gephyrocapsa muellerae. G. Emiliania huxleyi. H. Calcidiscus leptoporus. I. Helicosphaera carteri. J. Pontosphaera spp. K. Coccolithus pelagicus. L. Ceratolithus spp. M. Florisphaera profunda. N. Helicosphaera princei. O. Helicosphaera sellii. P. Helicosphaera wallichii. Q. Reticulofenestra pseudoumbilicus. R. Sphenolithus heteromorphus (lower) and Sphenolithus abies (higher). S-T. Discoaster variabilis. 
en menor medida por C. leptoporus (Figura 7). En cuanto a la especie $G$. oceanica, muestra una mayor correspondencia con el eje 2 , siendo el vector de este taxón el más cercano a dicho eje, mostrando preferencias por condiciones de mayor profundidad y de mayor afinidad bajo condiciones de alta influencia de sedimentos (Figura 7).

\section{DISCUSIÓN}

Comparación con otras asociaciones de cocolitóforos del Pacífico Tropical Oriental

El conteo de cocolitos indica que la asociación principal (mayor a 2\%) en las muestras de Chocó y Tumaco samples is: G. oceanica, G. muellerae, Gephyrocapsa $<3$ $\mu \mathrm{m}$, E. huxleyi, C.leptoporus and H.carteri (Figure 4; Table $2)$. The following were observed to a lesser extent $(<2 \%)$ : Ceratolithus spp., Coccolithus pelagicus, $F$. profunda, H. princei, H. sellii, H. wallichii and Pontosphaera spp. Together with reworked forms of $R$. pseudoumbilicus, $D$. variabilis, S. heteromorphus and S. abies (Figure 4). The dominant assemblage recorded in this study differs from the coccolithophores reported by Saavedra-Pellitero et al. (2010; 2011) near the Cocos and Carnegie Ridges (Figure 8). One of the most striking differences in this comparison is the number of specimens of $F$. profunda to the west in the Quaternary sediments of the Cocos Ridge, and to the south

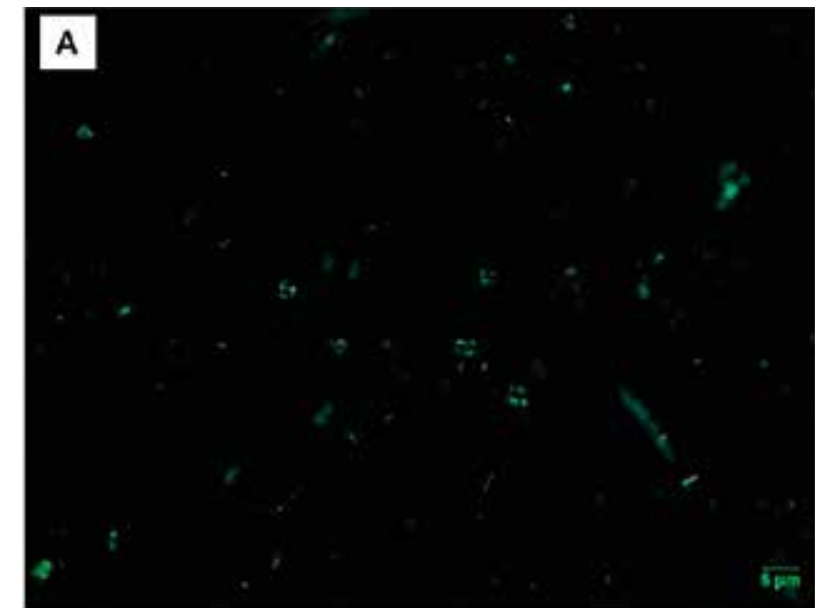

Figura 5. Ejemplo de la abundancia y preservación de los cocolitos. A. muestra PC-19-MER; B. muestra PC-15-EGO. Nótese la diferencia de abundancia y preservación en ambas muestras.

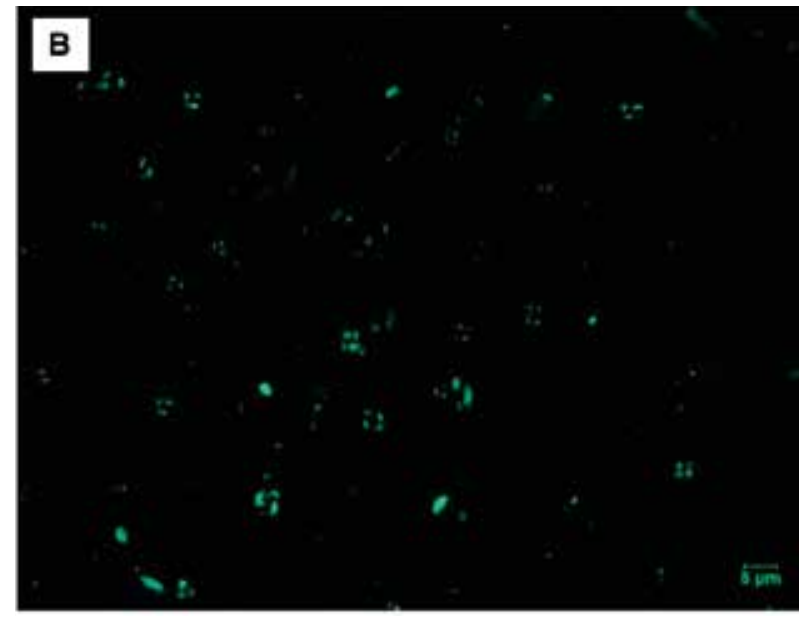

Figure 5. Example of the abundance and preservation of coccoliths. A. sample PC-19-MER; B. sample PC-15-EGO. Note the difference in abundance and preservation in the two samples. es G. oceanica, G. muellerae, Gephyrocapsa $<3 \mu \mathrm{m}, E$. huxleyi, C. leptoporus y H. carteri (Figura 4; Tabla 2). En una menor proporción $(<2 \%)$ se observaron Ceratolithus spp., Coccolithus pelagicus, $F$. profunda, $H$. princei, $H$. sellii, $H$. wallichii y Pontosphaera spp. junto con formas retrabajadas de $R$. pseudoumbilicus, D. variabilis, S. heteromorphus y $S$. abies (Figura 4). La asociación dominante registrada en este estudio difiere de los cocolitóforos registrados por Saavedra-Pellitero et al. $(2010,2011)$ cerca de la dorsal de Cocos y Carnegie (Figura 8). Una de las más marcadas diferencias en esta comparación es el número de ejemplares de $F$. profunda hacia el occidente en los sedimentos Cuaternarios de la Dorsal de Cocos y al sur en la Dorsal de Carnegie (Ahagon, 1993; Saavedra-Pellitero et al., 2010, 2011). in the Carnegie Ridge (Ahagon, 1993; Saavedra-Pellitero et al., 2010, 2011).

The most abundant species reported in the Cocos Ridge is $F$. profunda (34.89\%), followed by $G$. oceanica (21.98\%), Gephyrocapsa $<3 \mu \mathrm{m}(18.74 \%)$, and G. muellerae (10.75\%). In these assemblages, C. leptoporus, E. huxleyi and $H$. carteri present abundances of less than $4 \%$. The most abundant groups in the Carnegie Ridge continue to be F. profunda (28.8\%) and G. oceanica (26.2\%), followed by Gephyrocapsa <3 $\mu \mathrm{m}$ (16.1\%), E. huxleyi (11\%), G. muellerae (10.75\%) and C. leptoporus (4.3\%) (Figure $9)$, while this paper reports abundances of $G$. oceanica (24-26\%), G. muellerae (18-22\%), Gephyrocapsa $<3 \mu \mathrm{m}$ (14-15\%), E. huxleyi (12-14\%), C. leptoporus (3-4\%), H. 
Tabla 2. Especies de cocolitóforos con abundancias relativas $>2 \%$.

Table 2. Coccolithophores species with relative abundance $>2 \%$.

\begin{tabular}{|c|cc|}
\hline Especie & Cuenca Chocó & Cuenca Tumaco \\
Species & Chocó Basin & Tumaco Basin \\
\hline Gephyrocapsa oceanica & $26 \%$ & $24 \%$ \\
Gephyrocapsa muellerae & $18 \%$ & $22 \%$ \\
Gephyrocapsa $<3 \mu \mathrm{m}$ & $14 \%$ & $15 \%$ \\
Emiliania huxleyi & $12 \%$ & $14 \%$ \\
Calcidiscus leptoporus & $3 \%$ & $4 \%$ \\
Helicosphaera carteri & $3 \%$ & $2 \%$ \\
\hline
\end{tabular}
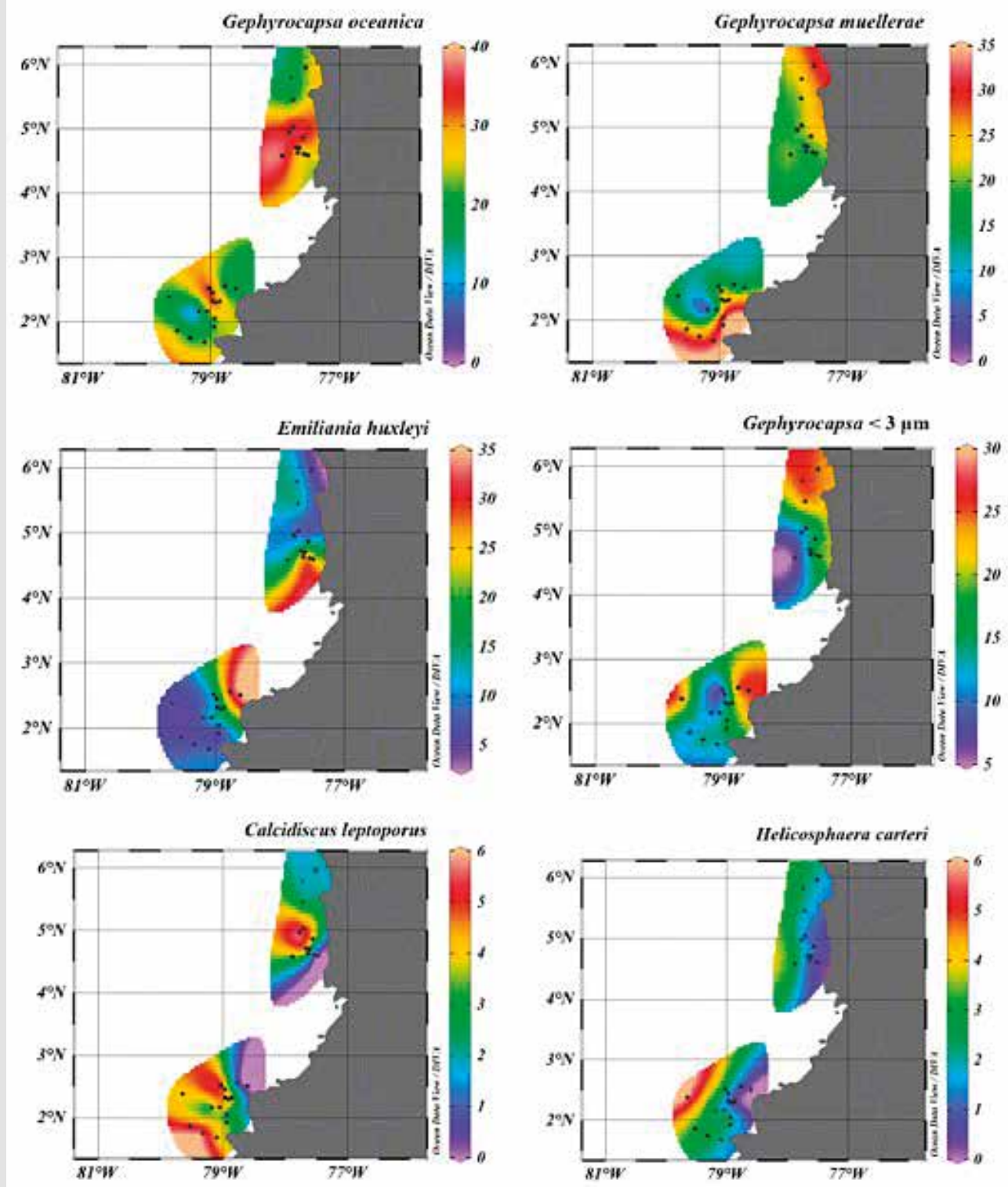

Figura 6. Mapas de distribución geográfica y abundancia relativa de las especies de cocolitóforos más abundantes ( $>2 \%)$ encontrados en los sedimentos.

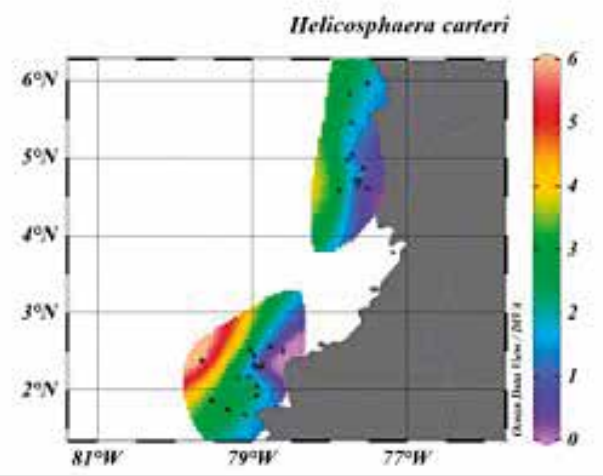

Figure 6. Geographic distribution and relative abundance maps of the most abundant $(>2 \%)$ coccolithophores species found in the sediments. 
En la Dorsal de Cocos la especie más abundante registrada es $F$. profunda $(34,89 \%)$, seguida de G. oceanica

$(21,98 \%)$, Gephyrocapsa $<3 \mu \mathrm{m}(18,74 \%)$ у $G$. muellerae $(10,75 \%)$. En dichas asociaciones, $C$. leptoporus,

$E$. huxleyi y $H$. carteri son componentes con abundancias inferiores a 4\%. En la Dorsal de Carnegie los grupos más abundantes continúan siendo $F$. profunda $(28,8 \%)$ y G. oceanica (26,2\%), seguidas de Gephyrocapsa $<3 \mu \mathrm{m}$ (16,1\%), E. huxleyi (11\%), G. muellerae $(10,75 \%)$ у C. leptoporus (4,3\%) (Figura 9), mientras que en este trabajo se registran abundancias de G. oceanica (24-26\%), G. muellerae (18-22\%), Gephyrocapsa $<3 \mu \mathrm{m}(14-15 \%)$, E. huxleyi (1214\%), C. leptoporus (3-4\%), H. carteri (2-3\%) y F. profunda con abundancias por debajo de $2 \%$ (Tabla 2). Estos patrones de distribución en los taxones podrían estar reflejando variaciones en las afinidades paleoecológicas de las especies y/o efectos de "sorting" asociados a la disolución. No obstante, discriminar entre los posibles factores que afectaron la distribución de las asociaciones requiere un estudio más exhaustivo que incluya otras herramientas químicas y paleontológicas junto con el uso de técnicas estadísticas. carteri (3-2\%), and F. profunda with abundances of below $2 \%$ (Table 2). These distribution patterns in taxa may reflect variations in the species' paleoecological affinities and/ or sorting effects associated with dissolution. However, discriminating between the possible factors that affected the distribution of assemblages requires a more comprehensive study that includes other chemical and paleontological tools along with the use of statistical techniques.

Abundance and preservation of coccoliths: Chocó vs. Tumaco

Descriptive and quantitative analyses of coccoliths assemblages extracted from the Chocó and Tumaco basins show a similarity in the composition of the nannoflora (Figure 4-6, Table 2). However, a difference was observed in the degree of preservation and abundance between the localities (Figure 5; Table 1). In the case of the Chocó Basin, its preservation was moderate and the number of coccoliths per gram was lower $\left(5.7 \times 10^{6} \mathrm{cc} / \mathrm{g}\right)$ compared to the Tumaco Basin. This value is related to the fact that the majority of

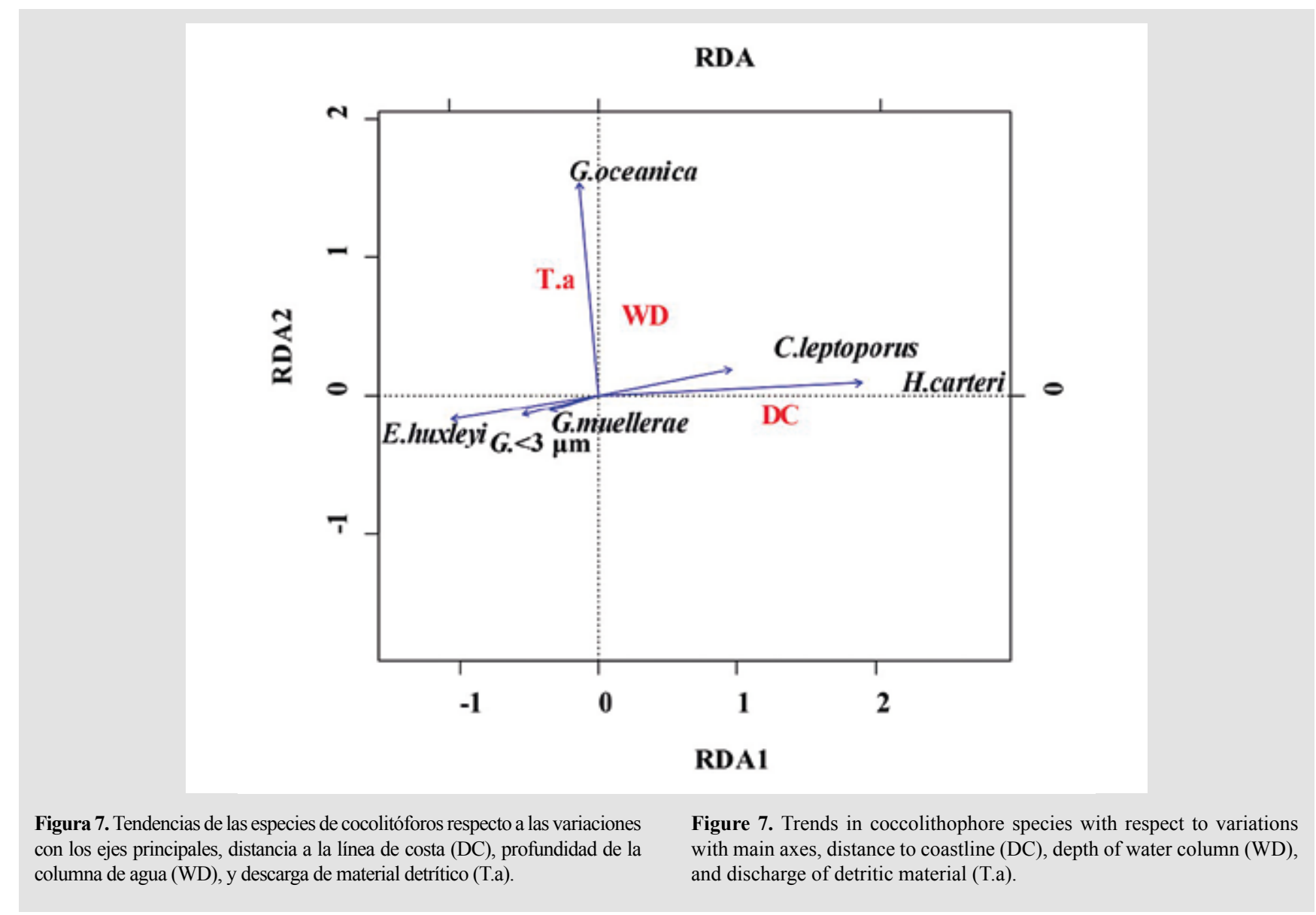


Tabla 3. Coeficiente de correlación entre las variables y los puntajes de las especies de cocolitos más representativas en el análisis de redundancia. Los valores en negrilla corresponden a los parámetros que presentaron mejor correlación con los ejes principales.
Table 3. Correlation coefficient between the variables and the scores of the most representative coccoliths species in the redundancy analysis. The values in bold correspond to the parameters that presented the best correlation with the main axes.

\begin{tabular}{|c|c|c|}
\hline Variable & Eje 1 & Eje 2 \\
Variable & Axis 1 2 \\
\hline $\begin{array}{c}\text { Profundidad / Depth } \\
\text { Distancia a la línea de costa / } \\
\text { Distance to coastline }\end{array}$ & 0.1615596 & 0.5770010 \\
Taxón & 1.4276028 & 0.2009410 \\
Descarga de sedimentos / Sediment discharge & -0.4538382 & 0.8374878 \\
Gephyrocapsa oceanica & -0.06829 & 0.75481 \\
Gephyrocapsa muellerae & -0.17191 & -0.05413 \\
Gephyrocapsa <3 $\mu \mathrm{m}$ & -0.27042 & -0.06642 \\
Emiliania huxleyi & -0.52305 & -0.08236 \\
Calcidiscus leptoporus & 0.47358 & 0.09479 \\
\hline
\end{tabular}

Abundancia y preservación de los cocolitos: Chocó vs Tumaco

El análisis descriptivo y cuantitativo de las asociaciones de cocolitos extraídos de las cuencas Chocó y Tumaco muestran una similitud en la composición de la nanoflora (Figura 4-6, Tabla 2). Sin embargo, se observó una diferencia en el grado de preservación y abundancia entre las localidades (Figura 5; Tabla 1). En el caso de la Cuenca Chocó, su preservación fue moderada y el número de cocolitos por gramo fue bajo $\left(5,7 \times 10^{6} \mathrm{cc} / \mathrm{g}\right)$ en comparación con la Cuenca Tumaco. Este valor se debe a que la mayoría de muestras presentaron abundancias muy bajas, 8 de 21 muestras fueron catalogadas como estériles y solamente 11 tuvieron un recobro superior a 100 cocolitos (Tabla 1). En contraste, para la Cuenca Tumaco sólo se obtuvo una muestra estéril, estimándose una buena preservación y un promedio de abundancia de $1,2 \times 10^{7}$ cc/g (Tabla 1). En un intento por explicar esta diferencia en abundancia y distribución, se realizó un análisis estadístico multivariado RDA evaluando las variables disponibles y que posiblemente controlaron la producción de cocolitos. Dicho análisis reveló que las fluctuaciones de las abundancias de los cocolitos $>2 \%$ pueden ser explicadas principalmente por la distancia a la línea de costa (Tabla 3; Figuras 6 y 7). Las mayores abundancias que se obtuvieron de E. huxleyi y Gephyrocapsa $<3 \mu \mathrm{m}$ se encontraron en las muestras cuya localización estuvo más cerca de la línea de costa y en zonas menos profundas (i.e. los puntos de muestreo PC-15 MER, PC-19 MER, PC-01-EGO y PC-03-EGO). Aunque $H$. carteri presentó bajas abundancias samples presented very low abundances, 8 of 21 samples were catalogued as barren, and only 11 had a recovery higher than 100 coccoliths (Table 1). In contrast, for the Tumaco Basin, only one barren sample was obtained, estimating good preservation and an average abundance of $1.2 \times 10^{7} \mathrm{cc} / \mathrm{g}$ (Table 1). In an attempt to explain this difference in abundance and distribution, a multivariate RDA statistical analysis was performed evaluating the available variables that may have controlled coccoliths production. This analysis revealed that fluctuations in coccoliths abundances $>2 \%$ can be explained mainly by distance from the coastline (Table 3; Figures $6 \mathrm{y}$ 7). The greatest abundances of E. huxleyi and Gephyrocapsa $<3 \mu \mathrm{m}$ were found in samples whose location was close to the coastline and in shallower areas (i.e., sampling points PC-15 MER, PC-19 MER, PC-01-EGO and PC-03-EGO). Although $H$. carteri showed low levels of abundance (Figure 6), the distribution and abundance of this species seem to be very controlled by the distance to the coastline as supported by the RDA analysis (Table 3) with the most abundance in the samples taken furthest from the continent (Figure 7). According to statistical processing, the variables with the lowest percentages of influence are: the amount of detritus per year and depth of the water column, these factors were not determinant in the abundance of coccoliths calculated for the samples examined.

Modern studies undertaken in the oceanic waters of the Chocó Basin indicate that the temperature is higher towards the coast, and higher compared to the temperature of the Tumaco Basin. In contrast, salinity is higher towards 
(Figura 6), la distribución y abundancia de esta especie parece estar muy controlada por la distancia a la línea de costa como lo sustenta el análisis RDA (Tabla 3), siendo más abundante en las muestras más alejadas del continente (Figura 7). Según el procesamiento estadístico, las variables con más bajos porcentajes de influencia son: la cantidad de detritos por año y profundidad de la columna de agua, estos factores no fueron determinantes en las abundancias de cocolitos calculadas para las muestras examinadas.

Modernos estudios en las aguas oceánicas de la Cuenca Chocó indican que la temperatura es mayor hacia la costa, y a su vez es mayor en comparación con la temperatura de la Cuenca Tumaco, en donde, por lo contrario, la salinidad es mayor hacia la zona distal y es más intensa que la presentada en the distal zone and is more intense than that presented in the waters of the Chocó Basin (Figure 2) (CCCP, 2002; NOAA, 2017). These characteristics are closely related to the influence of ocean currents of greater magnitude, such as the Chocó Counter Current (with less cold water) in the Chocó area, and the Peru Current (colder water masses) in areas of Tumaco (CCCP, 2002). Although in general these physical-chemical characteristics may be reflected by the microfossils found in this study, the lack of quantitative data does not allow an evaluation of the impact of these variables on the abundance and preservation of coccolithophore assemblages.

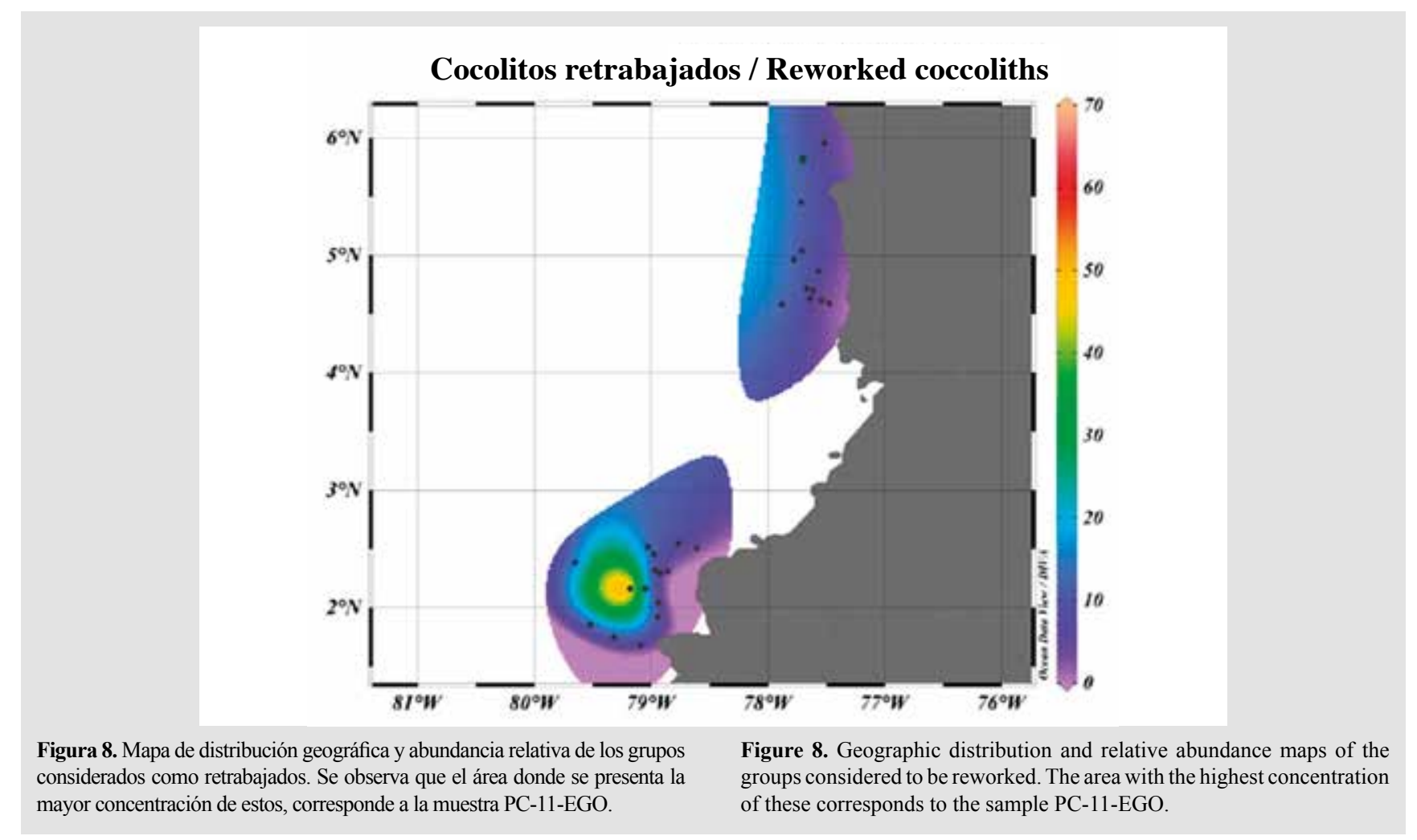

las aguas de la Cuenca Chocó (Figura 2) (CCCP, 2002; NOAA, 2017). Estas características se encuentran estrechamente relacionadas a la influencia de corrientes oceánicas de mayor magnitud, como la Contracorriente del Chocó (con aguas menos frías) en la zona del Chocó, y de la Corriente de Perú (masas de agua más frías) en áreas de Tumaco (CCCP, 2002). Aunque de forma general estas características físico-químicas pueden estar siendo reflejadas por los microfósiles hallados en este estudio, la falta de datos cuantitativos no permite realizar una

\section{Occurrence of reworking}

Reworked forms were identified and quantified among the results acquired by identifying and counting the coccoliths of the 39 samples (Figure 4 Q-T). The most common reworked species were those belonging to the genus Reticulofenestra spp. that was seen in most of the samples. The PC-11-EGO sample, located south of the Tumaco Basin, showed the highest content and variety of reworked species (Figures 3 and 9). 
evaluación del impacto de estas variables sobre la abundancia y preservación de las asociaciones de cocolitóforos.

Presencia de retrabajados

Dentro de los resultados adquiridos a través de la identificación y conteo de los cocolitos de las 39 muestras, fueron identificadas y cuantificadas las formas retrabajadas (Figura 4 Q-T). Los retrabajados más comunes fueron las especies pertenecientes al género Reticulofenestra, las cuales fueron vistas en la mayoría de las muestras. La muestra PC-11EGO, localizada al sur en la Cuenca Tumaco, mostró el mayor contenido y variedad de especies retrabajadas (Figuras 3 y 9).
Sphenolithus abies, Sphenolithus heteromorphus, Discoaster variabilis and Reticulofenestra spp. were the most abundant species in that sample (Figure 9). The presence of this assemblage in Pleistocene sediments enables us to infer the contribution of marine sediments from the MiocenePliocene to the study area. Based on the location of the PC11-EGO sample in the slopes of a positive relief (Figure 3), it is proposed that the origin of these calcareous nannofossils may be related to processes of seabed removal, which would incorporate forms of Neogene in Quaternary sedimentation. This seems to be supported by the fact that this was the only sample with retrieval of the oldest specimens, noticing that the

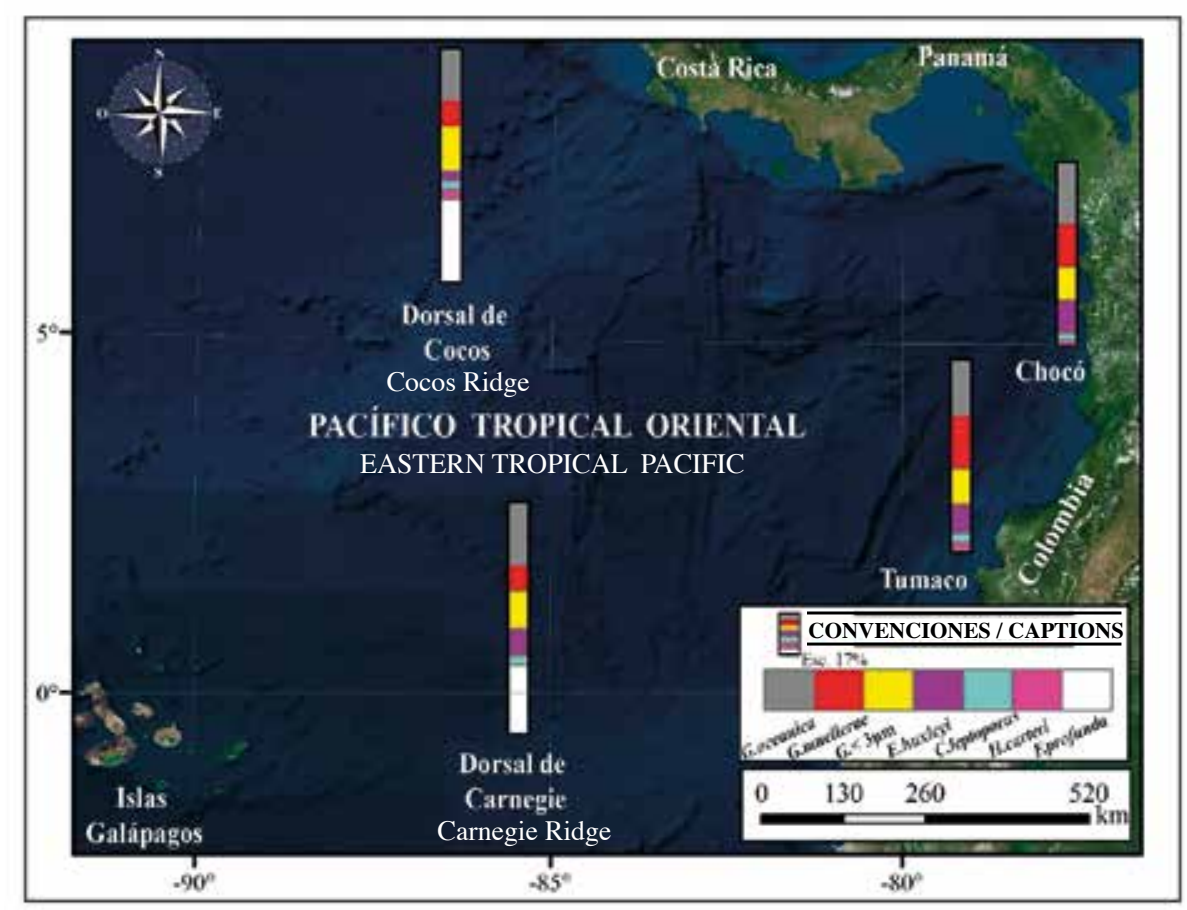

Figura 9. Comparación de las asociaciones y abundancias promedio de cocolitóforos reportadas en piston core tomados sobre la Dorsal de Cocos, la Dorsal de Carnegie (Saavedra-Pellitero 2010, 2011) y el Pacífico colombiano en este trabajo.
Figure 9. Comparison of the assemblages and average abundances of coccolithophores reported in piston core samples taken on the Cocos Ridge, the Carnegie Ridge (Saavedra-Pellitero 2010, 2011) and the Colombian Pacific in this work.
Sphenolithus abies, Sphenolithus heteromorphus, Discoaster variabilis, y Reticulofenestra spp. fueron las más abundantes en dicha muestra (Figura 9). La presencia de esta asociación en sedimentos del Pleistoceno permite inferir el aporte de sedimentos marinos del Mioceno-Plioceno al área de estudio. Con base en la localización de la muestra PC11-EGO en las vertientes de un relieve positivo (Figura 3), se propone que es factible que el origen de estos nanofósiles calcáreos pueda estar relacionado con procesos de remoción surrounding sites did not present forms of Sphenolithus and Discoaster. Additionally, although the course of the Mira and Patía rivers on the Neogene marine rocks exposed in Tumaco Bay may have constituted another origin of the reworked rocks (Borrero et al., 2012; Bedoya et al., 2013), the exclusive concentration of Miocene-Pliocene nannofossils in the PC-11EGO sample once again suggests more localized processes that would be providing older materials (Figure 3). 
del fondo marino, el cual incorporaría formas del Neógeno en la sedimentación Cuaternaria. Esto parece estar apoyado al hecho que ésta fue la única muestra con recuperación de los especímenes más antiguos, notándose que los sitios aledaños no presentaron formas de Sphenolithus y Discoaster. Adicionalmente, si bien el curso de los ríos Mira y Patía sobre las rocas Neógenas marinas expuestas en la Bahía de Tumaco pudieron haber sido otro origen de los retrabajados (Borrero et al., 2012; Bedoya et al., 2013), la concentración exclusiva de nanofósiles del Mioceno-Plioceno en la muestra PC-11-EGO sugieren una vez más procesos más localizados que estarían aportando materiales más antiguos (Figura 3).

\section{CONCLUSIONES}

Los análisis micropaleontológicos de 39 muestras extraídas de las Cuencas offshore de Tumaco y Chocó muestran que la asociación principal (mayor al 2\%) de cocolitóforos es G. oceanica, G. muellerae, Gephyrocapsa $<3 \mu \mathrm{m}$, E. huxleyi, C. leptoporus y H. carteri. En una menor proporción $(<2 \%)$ se observaron Ceratolithus spp., C. pelagicus, F. profunda, $H$. princei, $H$. sellii, $H$. wallichii y Pontosphaera spp. junto con formas retrabajadas de Reticulofenestra spp, Sphenolithus spp. y Discoaster spp. del Mioceno-Plioceno. La presencia de $E$. huxleyi dentro de la asociación indica que la edad de estos sedimentos no debe ser más antigua que la biozona NN21 del Ioniano (Pleistoceno Medio). Las estimaciones del número de cocolitos por gramo (cc/g) revelaron que la abundancia promedio de la Cuenca Chocó $\left(5,7 \times 10^{6} \mathrm{cc} / \mathrm{g}\right)$ es menor en comparación con la Cuenca Tumaco $\left(1,2 \times 10^{7} \mathrm{cc} / \mathrm{g}\right)$. De acuerdo con los análisis estadísticos multivariados RDA, esta diferencia puede ser explicada principalmente por la distancia a la costa, la cual parece haber controlado la distribución y abundancia de los cocolitos encontrados.

\section{AGRADECIMIENTOS}

Los autores agradecemos al geólogo Eduardo López, de la Vicepresidencia de Exploración de Ecopetrol S.A por la descripción de los core, y al tecnólogo Adrián Delgado por los muestreos llevados a bordo del ARC Providencia. Igualmente, deseamos agradecer al comandante Ferrero, a la tripulación del ARC Providencia y al grupo de profesionales de la compañía GEMS quienes manejaron la adquisición de los piston core. Especiales agradecimientos a Valentina Ramírez Valencia por sus valiosos aportes en los análisis estadísticos, y también a la Profesora Gladys Bernal y los revisores anónimos por su contribución al mejoramiento del manuscrito y su publicación.

\section{CONCLUSIONS}

Micro paleontological analyses of 39 samples taken from the offshore Tumaco and Chocó Basins show that the main assemblage (greater than 2\%) of coccolithophores is G. oceanica, G. muellerae, Gephyrocapsa $<3 \mu \mathrm{m}, E$. huxleyi, $C$. leptoporus and H.carteri. In a smaller proportion (<2\%) Ceratolithus spp., C. pelagicus, F. profunda, H. princei, H. sellii, H. wallichii and Pontosphaera spp. were observed along with reworked forms of Reticulofenestra spp., Sphenolithus spp. and Discoaster spp. of MiocenePliocene. The presence of E. huxleyi within the assemblage indicates that the age of these sediments should not be older than Ionian biozone NN21 (Middle Pleistocene). Estimates of the number of coccoliths per gram $(\mathrm{cc} / \mathrm{g})$ revealed that the average abundance of the Chocó Basin $\left(5.7 \times 10^{6} \mathrm{cc} / \mathrm{g}\right)$ is lower compared to the Tumaco Basin $\left(1.2 \times 10^{7} \mathrm{cc} / \mathrm{g}\right)$. According to RDA multivariate statistical analyses, this difference can be explained mainly by distance to the coast, which seems to have controlled the distribution and abundance of the coccoliths found.

\section{ACKNOWLEDGEMENTS}

The authors are grateful to geologist Eduardo López, from the Vice-presidency of Exploration at Ecopetrol S.A for the description of the core, and to technologist Adrián Delgado for the samples taken aboard the ARC Providencia. We would also like to thank Commander Ferrero, the crew of ARC Providencia and the group of GEMS professionals who managed the acquisition of the piston core. Special thanks to Valentina Ramírez Valencia for her valuable contributions in the statistical analyses, and to Professor Gladys Bernal and the anonymous reviewers for their contribution to the manuscript. 


\section{BIBLIOGRAFÍA / LITERATURE CITED}

Ahagon, N., Y. Tanaka and H. Ujiie. 1993. Florisphaera profunda, a possible nannoplankton indicator of late Quaternary changes in sea-water turbidity at the northwestern margin of the Pacific. Mar. Micropaleontol., 22: 255-273.

Antunes, R. L. 2007. Nanofósseis calcários do Quartenário da margem continental brasileira. Petrobrás, p. 75.

Backman, J., I. Raffi, D. Rio, E. Fornaciari and H. Pälike. 2012. Biozonation and biochronology of Miocene through Pleistocene calcareous nannofossils from low and middle latitudes. Newslet. Stratigr., 45 (3): 221-244.

Balch, W. M. 2004. Re-evaluation of the physiological ecology of coccolithophores: 165-190. In Coccolithophores, Springer, Berlin, Heidelberg.

Bedoya, E. L., J.A. Flores y A. Pardo. 2013. Nanofósiles calcáreos y bioestratigrafía del Mioceno Tardío del SW de la Cuenca Tumaco onshore (Pacífico Colombiano). Bol. Geol. UIS, 35(1): 55-66.

Betancur, M. J. e I. Martínez. 2003. Foraminíferos bentónicos recientes en sedimentos de fondo de la Cuenca de Panamá (Pacífico Colombiano), como indicadores de productividad y oxigenación. Bol. Invest. Mar. Cost., 32: 92-123.

Borcard, D., F. Gillet and P. Legendre. 2011. Numerical ecology with R. Springer, New York, p 435.

Borrero, C., A. Pardo, C.M. Jaramillo, J.A. Osorio, J.A. Cardona, J-A. Flores, J-A., S. Echeverry Rosero, J. García y H. Castillo. 2012. Tectonoestratigraphy of the Cenozoic Tumaco forearc basin (Colombian Pacific) and this relationship with the northern Andes orogenic build up. J. South Am. Earth Sci., 39 : 75-92.

Bown, P. R. 1998. Calcareous nannofossil biostratigraphy: 315. Bown, P. (Ed.). Chapman and Hall.

Centro Control Contaminación del Pacífico CCCP. 2002. Compilación oceanográfica de la cuenca Pacífica colombiana. Serie Publ. Esp. CCCP, 107 p.

D'Croz, L. and A. O'Dea. 2007. Variability in upwelling along the Pacific Shelf of Panama and implications for the distribution of nutrients and chlorophyll. Est., Coast. Shelf Sci., 73: 325-340.

D'Croz, L. and A. O’Dea. 2009. Nutrient and chlorophyll dynamics in Pacific Central America (Panama): 335-344. In: Lang, M.A., I.G. MacIntyre and K. Ruetzler (Eds.), Proc. Smithsonian Mar. Sci. Symp. Smithsonian Institution Scholarly Press, Washington DC.

Dennison, J. M. and W.W. Hay. 1967. Estimating the needed sampling area for subaquatic ecological studies. J. Paleontol., 4: 706-708.

Fatela, F. and R. Taborda. 2001. Confidence limits of species proportions in microfossil assemblages. Mar. Micropaleontol., 45: 169-174.

Fiedler, P. C. and L.D. Talley. 2006. Hydrography of the eastern tropical Pacific: A review. Progr. Oceanogr., 69 (2): 143-180.

Flores, J. A. and F.J. Sierro. 1997. Revised technique for calculation of calcareous nannofossil accumulation rates. Micropaleontology, 43 : 321-324.

Flores, J.A. y F.J. Sierro. 2007. Paleoceanography, biological proxies Coccoliths. Elsevier, Oxford.1646 p.

Flores, J. A., F.J. Sierro, G. Francés, A. Vázquez, A. and I. Zamarreño. 1997. The last 100,000 years in the Western Mediterranean: sea surface water and frontal dynamics as revealed by coccolithophores. Mar. Micropaleontol., 29: 351-366.

Flores, J. A., R. Gersonde y F.J. Sierro. 1999. Pleistocene fluctuations in the Agulhas Current Retroflection based on the calcareous plankton record. Mar. Micropaleontol., 37: 1-22.

Flores, J.A., M.A. Bárcena and F.J. Sierro. 2000. Ocean-surface and wind dynamics in the Atlantic Ocean off Northwest Africa during the last 140000 years. Palaeogeogr., Palaeoclimatol., Palaeoecol., 161: 459-478.

Giraudeau, J. 1992. Distribution of recent nannofossils beneath the Benguela system: southwest African continental margin. Mar. Geol., 108: $219-237$.

Gómez, C. e I. Martinez. 2005. Nueva productividad biológica en el Pacífico oriental ecuatorial: respuesta al aporte de nutrientes por los ríos del Pacífico colombiano, surgencia costera y polvo atmosférico. Bol. Geol., 27 (2): 100-114.

Hammer, Ø., D. Harper and P. Ryan. 2001. Paleontological statistics software: package for education and data analysis. Palaeontol. Electron., 4: 1-9.

Jordan, R. W., A. Kleijne, B.R. Heimdal and J.C. Green. 1995. A glossary of the extant Haptophyta of the world. J. Mar. Biol. Ass. UK, 75 (4): $769-814$.

Knauss, J. A. 1960. Measurements of the Cromwell Current. Deep Sea Res., 6: 265-286.

López, J. C. R. y P. Costeros. 2006. Aporte de caudales de los ríos Baudó, San Juan, Patía y Mira a la cuenca Pacífica colombiana. Bol. Cient. CCCP, 13: 17-32.

Málikov, I. y N. Villegas. 2005. Construcción de series de tiempo de temperatura superficial del mar de las zonas homogéneas del océano Pacífico colombiano. Bol. Cient. CCCP, 12: 79-93.

Martini, E. 1971. Standard Tertiary and Quaternary calcareous nannoplankton zonation: 739-785. In: Farinacci, A. (Ed.) Proceedings of the Second Planktonic Conference, Rome, 2. Tecnosciencia, Rome.

McIntyre, A. and A.W.H. Bé. 1967. Modern Coccolithophoridae of the Atlantic Ocean I. Placoliths and Cyrtoliths. Deep-Sea Res., 14: $561-597$.

McIntyre, A., A.W.H. Bé and M.B. Roche. 1970. Modern Pacific Coccolithophorida: a paleontological thermometer. Transact. NY Acad. Sci. Ser. II, $32: 720-731$.

Okada, H. 1983. Modern nannofossil assemblages

Okada, H. and S. Honjo. 1973. The distribution of oceanic coccolithophores in the Pacific. Deep-Sea Res., 20: 355-374.

Okada, H. and A. McIntyre. 1979. Seasonal distribution of modern coccolithophores in the western North Atlantic Ocean. Mar. Biol., 54: $319-328$.

Pak, P., Jr. and V. Zaneveld. 1974. Equatorial front in the Eastern Pacific Ocean. J. Phys. Oceanogr., 4: 570-578. 
Patarroyo, G. D. y J.I. Martínez. 2013. Foraminíferos bentónicos recientes en las aguas profundas de la Cuenca de Panamá: ecología y su posible relación con las corrientes de fondo. Bol. Invest. Mar. Cost., 42 (1): 31-55.

Patarroyo, G. D. y J.I. Martínez. 2016. Paleoxigenación y paleoproductividad en el Golfo de Panamá durante el Holoceno tardío. Bol. Geol., 38 (2): $75-92$.

Perch-Nielsen, K. 1985. Cenozoic calcareus nannofossils: 329-426. In: H. M. Bolli, J. B. Saunders and K. Perch-Nielsen (Eds.): Plankton stratigrafphy. Cambridge University Press, Cambridge.

Poulton, A. J., P.M. Holligan, A. Charalampopoulou and T.R. Adey. 2017. Coccolithophore ecology in the tropical and subtropical Atlantic Ocean: New perspectives from the Atlantic meridional transect (AMT) program. Progr. Oceanogr., 158: 150-170.

Poveda, G. and J. Mesa. 2000. On the existence of Lloró (the rainiest locality on Earth): enhanced ocean-land- atmosphere interaction by a low-level jet. Geophys. Res. Let., 27: 1675-1678.

Raffi, I. and J.A. Flores. 1995. Pleistocene through Miocene calcareous nannofossils from Eastern Equatorial Pacific Ocean (Leg 138): 233-286. In: N.G. Pisias, L.A., T.R. Mayer, A. Janecek, A. Palmer-Julson and T.H. van Andel (Eds.) Proceedings of the Ocean Drilling Program, Scientific Results, 138. College Station, US.

Restrepo, J. 2006. Aporte de caudales de los ríos Baudó, San Juan, Patía y Mira a la Cuenca Pacífica colombiana. Bol. Cient. CCCP, (13): 17-32.

Restrepo, J. D. e I.D. Correa. 2002. Geología y oceanografía del delta del río San Juan, litoral Pacífico colombiano. Universidad EAFIT, Medellín. 132 p. Restrepo, J. D. and S.A. López. 2008. Morphodynamics of the Pacific and Caribbean deltas of Colombia, South America. J. South Am. Earth Sci., $25:$ 1-21.

Restrepo, J.D., B. Kjerfve, I.D. Correa and J. González. 2000. Morphodynamics of a high discharge tropical delta, San Juan River, Pacific coast of Colombia. Mar. Geol., 192 (4): 355-381.

Rodríguez-Rubio, E.J., D.M. Guerrero y J.G. Rueda-Bayona. 2008. Caracterización oceanográfica de las corrientes costeras del Pacífico colombiano (Corriente Colombia-Corriente del Chocó), una revisión actualizada. Grupo Est. Oceanogr. Fen. El Niño. Área Oceanogr. Operac., Centro Control Contam. Pacífico (CCCP-DIMAR). Tumaco, Colombia. 2 p.

Roth, P. H. 1983. Jurassic and Lower Cretaceous calcareous nannofossils in the western north-Atlantic (site- 534)-biostratigraphy, preservation, and some observations on biogeography and paleoceanography. Init. Rep. Deep Sea Drill. Proj., 76: 587.

Roth, P. H. and H.R. Thierstein. 1972. Calcareous nannoplankton: Leg 14. Deep Sea Drilling Project.

Saavedra-Pellitero, M., J.A. Flores, K.H. Baumann and F.J. Sierro. 2010. Coccolith distribution patterns in surface sediments of Equatorial and Southeastern Pacific Ocean. Geobios, 43 (1): 131-149.

Saavedra-Pellitero, M., J.A. Flores, F. Lamy, F.J. Sierro and A. Cortina. 2011. Coccolithophore estimates of paleotemperature and paleoproductivity changes in the southeast Pacific over the past 27 kyr. Paleoceanography, 26: 1-16.

Schneider, L. J., T.J. Bralower and L.R. Kump. 2011. Response of nannoplankton to early Eocene ocean destratification. Palaeogeogr., Palaeoclimatol., Palaeoecol., 310 (3-4): 152-162.

Thierstein, H.R. and J.R. Young. 2004. Coccolithophores: from molecular processes to global impact: Springer Media Verlag, Berlin.

Thierstein, H.R., K.R. Geitznauer, B. Molfino and N.J. Shackleton. 1977. Global synchronicity of late Quaternary coccolith datum levels: validation by oxygen isotopes. Geology, 5: 400-404.

Whitlock, M. C. and D. Schluter. 2009. The analysis of biological data. Roberts, Greenwood Village, USA.

Wyrtki, K. 1965. Corrientes superficiales del océano Pacífico Oriental Tropical. Comisión Interamericana del Atún Tropical, 9: 295-304, p 565.

Wyrtki, K. 1966. Oceanography of the Eastern Equatorial Pacific Ocean. Mar. Biol. An. Rev., 4: 33-68, p 704.

Young, J. R., M. Geisen, L. Cros, A. Kleijne, C. Sprengel, I. Probert and J. Østergaard. 2003. A guide to extant coccolithophore taxonomy. J. Nannoplank. Res., 125.

Zar, J. 1996. Biostatistical analysis. Prentice Hall, New Jersey. 940 p.

\section{Referencias Virtuales / Digital references}

CIIFEN http://ciifen.org/index.php INA http://ina.tmsoc.org/

Nannotax3 http://www.mikrotax.org/Nannotax3/

NASA (Visible Earth) http://www.visibleearth.nasa.gov/

NOAA https://www.esrl.noaa.gov/ 


\section{APÉNDICE A. / APPENDIX A.}

A continuación, se presentan los taxones de cocolitóforos que fueron identificados en esta investigación. The following are the coccolithophore taxa that were identified in this study.

Calcidiscus leptoporus (Murray y Blackman, 1898) Loeblich y Tappan, 1978.

Ceratolithus cristatus (Kamptner, 1950).

Coccolithus pelagicus (Wallich, 1877) Schiller, 1930.

Discoaster brouweri (Tan, 1927) emend. Bramlette y Riedel, 1954.

Discoaster pentaradiatus (Tan, 1927).

Discoaster variabilis (Martini y Bramlette, 1963).

Emiliania huxleyi (Lohmann, 1902) Hay y Mohler en Hay et al., 1967.

Florisphaera profunda (Okada y Honjo, 1973) var. profunda Okada y McIntyre, 1979.

Gephyrocapsa muellerae (Bréhéret, 1978).

Gephyrocapsa oceanica (Kamptner, 1943) McIntyre y Bé, 1967; McIntyre et al. 1970.

Helicosphaera carteri (Wallich, 1877) Kamptner, 1954 var. carteri. McIntyre y Bé, 1967.

Helicosphaera princei (da Gama y Varol, 2013).

Helicosphaera sellii (Bukry y Bramlette, 1969) Jafar y Martini, 1975.

Helicosphaera wallichii (Lohmann, 1902) Okada y McIntyre, 1979.

Pontosphaera spp. (Kamptner, 1948) exDeflandre in Deflandre y Fert, 1954; Roth, 1970.

Reticulofenestra spp. (Hay et al., 1966).

Sphenolithus abies (Deflandre in Deflandre y Fert, 1954).

Sphenolithus heteromorphus (Deflandre, 1953). 


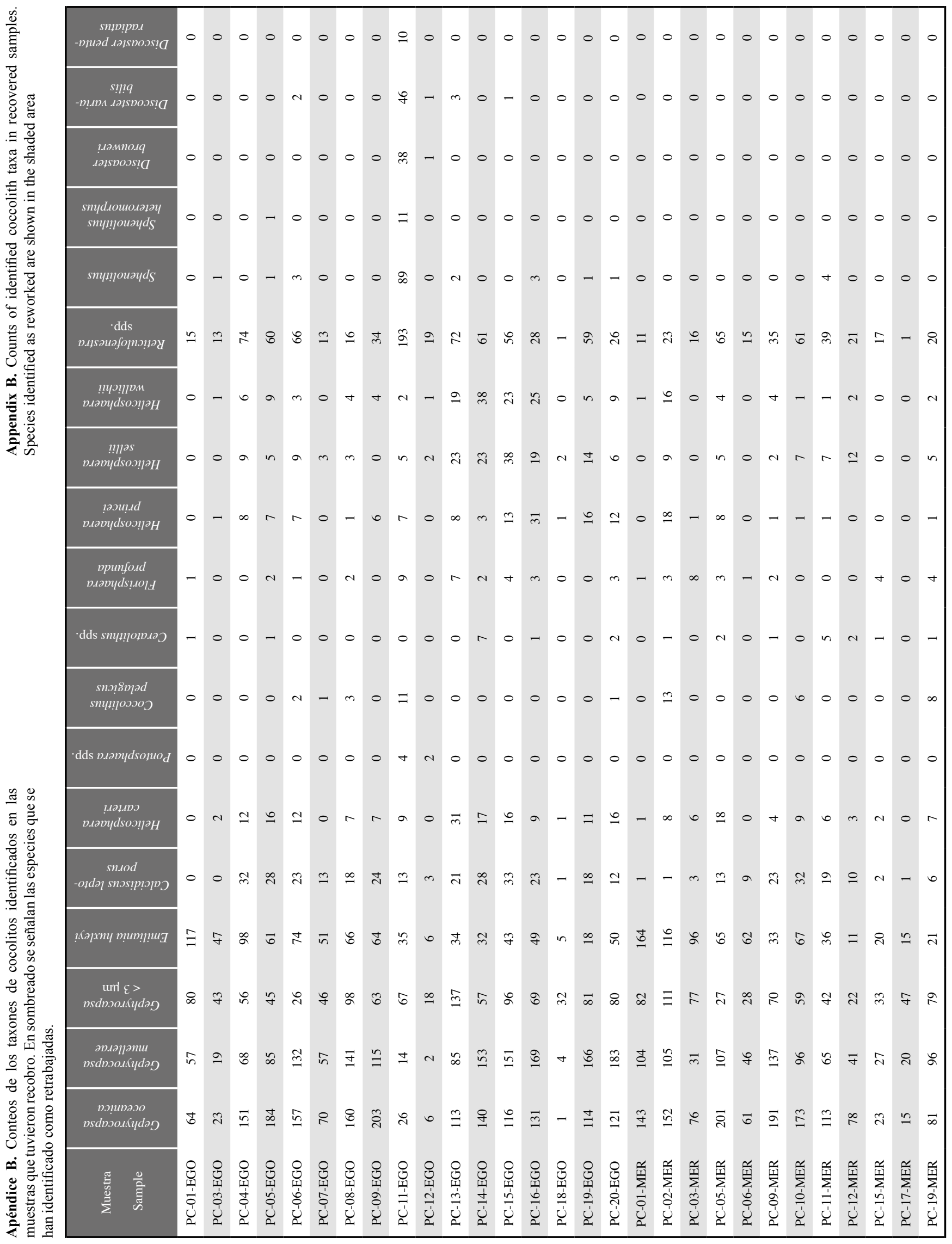

